\title{
Colicin E1 opens its hinge to plug TolC
}

\section{Authors}

S. Jimmy Budiardjo ${ }^{1}$, Jacqueline J. Stevens ${ }^{2}$, Anna L. Calkins ${ }^{3}$, Ayotunde P. Ikujuni ${ }^{2}$, Virangika K. Wimalasena ${ }^{2}$, Emre Firlar ${ }^{4}$, David A. Case ${ }^{5}$, Julie S. Biteen ${ }^{3}$, Jason T. Kaelber ${ }^{4}$ and Joanna S.G. Slusky ${ }^{1,2, *}$

\section{Affiliations}

${ }^{1}$ Center for Computational Biology, The University of Kansas, 2030 Becker Dr., Lawrence, KS 66047.

${ }^{2}$ Department of Molecular Biosciences, The University of Kansas, 1200 Sunnyside Ave. Lawrence KS 66045.

${ }^{3}$ Department of Chemistry, University of Michigan, Ann Arbor MI 48109-1055.

${ }^{4}$ Rutgers CryoEM \& Nanoimaging Facility and Institute for Quantitative Biomedicine, Rutgers University, Piscataway, NJ 08854, USA.

${ }^{5}$ Department of Chemistry and Chemical Biology, Rutgers University, Piscataway, NJ 08854 USA.

*Correspondence: slusky@ku.edu.

\begin{abstract}
The double membrane architecture of Gram-negative bacteria forms a barrier that is impermeable to most extracellular threats. Bacteriocin proteins evolved to exploit the accessible, surface-exposed proteins embedded in the outer membrane to deliver cytotoxic cargo. Colicin E1 is a bacteriocin produced by, and lethal to, Escherichia coli that hijacks the outer membrane proteins TolC and BtuB to enter the cell. Here we capture the colicin E1 translocation domain inside its membrane receptor, TolC, by high-resolution cryoEM to obtain the first reported structure of a bacteriocin bound to TolC. Colicin E1 binds stably to TolC as an open hinge through the TolC pore - an architectural rearrangement from colicin E1's unbound conformation.
\end{abstract}


This binding is stable in live $E$. coli cells as indicated by single-molecule fluorescence

microscopy. Finally, colicin E1 fragments binding to TolC plug the channel, inhibiting its native efflux function as an antibiotic efflux pump and heightening susceptibility to three antibiotic inhibit other outer membrane protein functions.

\section{Introduction}

In Gram-negative bacteria, the outer membrane, cell wall, and cytoplasmic membrane form concentric barriers that protect the cell from extracellular threats. Of these protective structures, the outer membrane forms a particularly formidable obstacle (Alexander \& Rietschel, 2001), owing to the impermeability of the lipopolysaccharide (LPS) layer that constitutes the outer membrane (Kamio \& Nikaido, 1976). The primary means by which external molecules can gain access to the cell is through the $\sim 100$ variations of barrel-shaped proteins that are embedded in each bacterium outer membrane (Freeman \& Wimley, 2012) and whose diverse functions include the transport of molecules across the membrane-specifically, the import of nutrients and metabolites and the export of toxins and waste.

Because outer membrane proteins (OMPs) are accessible from outside the cell, bacteriophage and bacterial toxins have evolved to exploit OMPs to initiate delivering cargo across the outer membrane. Bacteriocins hijack the OMPs of a target bacterium to cross its impermeable outer membrane and kill the bacterium. Colicins are E. coli-specific bacteriocins, protein toxin systems through which bacteria engage in bacterial warfare with other, similar bacteria. Colicins can be divided into groups A and B based on the requirement for which periplasmic protein systems they rely on to enter cells. The cytotoxicity of group A colicins requires the Tol system (Nagel de Zwaig \& Luria, 1967) which is coupled to the proton motive force for its role in maintaining membrane integrity (Lazzaroni et al., 1989). Colicins differ in 
their receptor targets and killing mechanisms. Most colicins share a common tri-domain architecture, comprising the following components: (i) an N-terminal translocation (T) domain, (ii) a receptor-binding (R) domain, and (iii) a C-terminal cytotoxic (C) domain (Figure 1A). Much of what is known of group A colicin import has been determined through studies of the colicin E3 and E9 as reviewed by Cramer et al. (Cramer et al., 2018). For colicins E3 and E9 import is initialized by high affinity R domain binding to the vitamin B12 transporter, BtuB (Kurisu et al., 2003, Housden et al., 2005, Francis et al., 2021); this binding localizes the colicin onto the outer membrane. Once the colicin is tethered to the outer membrane surface, the T domain of ColE3 and ColE9 initiates translocation using the secondary OMP receptor/translocator OmpF to access the Tol-Pal system in the periplasm. For most colicins, the T domain requires an OMP distinct from the $\mathrm{R}$ domain target for colicin translocation (Cascales et al., 2007). A handful of colicins have been structurally characterized with their OMP counterparts (Kurisu et al., 2003, Sharma et al., 2007, Buchanan et al., 2007). Colicin E9 bound to both BtuB and OmpF was structurally resolved as a complete translocon complex (Francis et al., 2021). The structure of the full ColE9 translocon showed that $\mathrm{T}$ domain can fully penetrate deeply into lumen of the outer membrane receptors while the $\mathrm{R}$ domain interact with the extracellular loop regions of its receptors. Studies of the bacteriocin pyocin S5 from Pseudomonas aeruginosa suggest that bacteriocin architectures and mechanisms may be conserved across all Gram-negative species (Behrens et al., 2020).

Colicin E1 is a bacteriocin produced by $E$. coli that enters the periplasm of neighboring cells and forms a pore on the cytoplasmic membrane leading to membrane depolarization and cell death. Unlike any of the other E colicins, the T domain of Colicin E1 binds to TolC (Benedetti et al., 1991), the outer membrane component of the acridine efflux pump, and the R domain binds BtuB (Benedetti et al., 1989) the primary receptor shared by all E colicins. A high-resolution xray structure exists of only the cytotoxic domain of colicin E1 (Elkins et al., 1997) but not the T or R domains, thus no mechanistic insights can be gained about initial steps of import. Domain 
87 swapping experiments (Benedetti et al., 1991) between colicin A and E1 identified regions of the

protein that interact with specific outer membrane proteins. Early studies of pore forming colicins, including E1, showed that cytotoxic-domain-induced $\mathrm{K}^{+}$efflux on the inner membrane can be reversed after it has begun by subsequent addition of trypsin to cells. This, in addition to trypsin's inability to cross the outer membrane, led to the belief that portions of colicin remain tethered to their OMP partners as the cytotoxic domain depolarizes the cytoplasmic membrane.(Plate \& Luria, 1972, Dankert et al., 1980, Dankert et al., 1982, Benedetti et al., 1992)

In order to understand the early stages of colicin E1 import, functional studies of truncations of colicin E1 which lack the cytotoxic domain have been characterized in vitro. Residues 100-120 of colicin E1 (termed the 'TolC box', Figure 1A) have been shown to be necessary but not sufficient for binding TolC. Peptides that include the TolC box co-elute with TolC (Zakharov et al., 2016) and disrupt channel conductance in planar lipid bilayer membranes(Zakharov et al., 2004, Zakharov et al., 2016). Moreover, E. coli exposure to TolCbox-containing peptides can prevent subsequent binding and cytotoxicity of full-length colicin E1 (Jakes, 2017). Circular dichroism of the T domain indicates that it exists as a helical hairpin (closed hinge) in solution similar to other colicin T domains (Wiener et al., 1997, Housden et al., 2021) and that the proline at the center of the TolC box forms its apex (Zakharov et al., 2016, Housden et al., 2021). This measurement led to the proposal, known as the "pillar model," (Zakharov et al., 2016) that the T domain inserts into the TolC barrel as a helical hairpin where the $\mathrm{N}$ and $\mathrm{C}$-termini are pointing to the cell exterior. According to this model, the hairpin stuck in TolC acts as a buttress to facilitate the $\mathrm{C}$ terminal-first cytotoxic domain entry directly through the membrane where it is possibly cleaved from the TR domain to reach its inner membrane target similar to ColE7 (Shi et al., 2005) and ColD (de Zamaroczy et al., 2001).

A competing model for colicin E1 import, known as the "total thread" model (Cramer et al., 2018, Zakharov et al., 2016, Housden et al., 2013, Francis et al., 2021) posits that the protein 
112 unfolds and passes through TolC N-terminus-first as an unstructured peptide and binds to TolA

113 (Pilsl \& Braun, 1995) in the periplasm. In this model the binding between the intrinsically

114 unstructured colicin N-termini and periplasmic proteins (Jakes, 2017, Housden et al., 2013)

115 creates a pulling force that results in the translocation of the whole colicin.

116 Here we use cryogenic electron microscopy (cryoEM) to solve the high-resolution

117 structure of a bacteriocin, colicin E1, bound to TolC. We find that colicin E1 binds stably to TolC,

118 not as a helical hairpin but as a single-pass folded helix with the $\mathrm{N}$-terminus inside the periplasm.

119 Additionally, we find that the ColE1 TR domain binds TolC in vivo as well. Using single-

120 molecule fluorescence microscopy, we find that ColE1-TR, lacking the cytotoxic domain,

121 remains stalled on the outer membrane and does not fully translocate into cells. Lastly, we

122 leverage this stalling of ColE1 to block the native TolC function as an antibiotic efflux pump.

123 Because they are accessible from outside the cell, OMPs are attractive targets for the development

124 of novel antibiotics, and research has begun to reveal the therapeutic potential of interfering with

125 OMP structure and function. (Storek et al., 2018, Kaur et al., 2021)

126 Here by determining the structure and mechanism of ColE1 insertion we establish an

127 alternative approach for targeting OMPs - the development of molecular plugs that block OMP

128 pores. Such plugs would allow for the manipulation of bacterial transport, providing a means of

129 either starving the bacterium by preventing the import of valuable nutrients or poisoning the

130 bacterium by preventing the export of toxins. Through real-time efflux assays and minimum

131 inhibitory concentration (MIC) experiments, we find that ColE1-TR and ColE1-T are able to

132 inhibit TolC-mediated efflux. We find that this plugging of TolC reduces the amount of

133 antibiotics required to inhibit the bacterial growth — indicating that this colicin E1 fragment

134 reduces the antibiotic resistance conferred by TolC. 


\section{Results}

\section{CryoEM structure of TolC embedded in nanodiscs}

To determine the structural details of colicin E1 binding to TolC, we solved the cryoEM (cryogenic electron microscopy) structure of TolC embedded in nanodiscs with and without added ColE1-T (PDB 6WXH and 6WXI, respectively). We recently reported a high-yield TolC preparation by refolding from inclusion bodies (Budiardjo et al., 2021) and inserted refolded TolC into nanodiscs. The cryoEM structure of refolded TolC alone (Figure 1B) is similar to the previously published crystal structures of natively derived TolC alone (Koronakis et al., 2000) or in complex with hexamminecobalt (Higgins et al., 2004) but with more splayed loops at the periplasmic opening (residues 165-175). There was local variation in resolution within the model with lower resolution associated with the extracellular/periplasmic ends and the nanodisc scaffold protein (Figure 1B). The lower residue resolution at these apertures may indicate dynamics not captured in the x-ray crystal structures.

We next determined binding of colicin E1-T domain to capture the complex for structure determination. Residues 1-190 (ColE1-T) which span the translocation domain are known to bind to TolC (Figure 1A). We identified ColE1-T:TolC binding in vitro via co-elution by size exclusion chromatography (SEC) as previously described (Zakharov et al., 2016). When ColE1-T and TolC were mixed, we observed a leftward shift in the TolC peak and a decrease in intensity associated with the ColE1-T peak indicating that a subset of the population had migrated with TolC (Figure 1C). We analyzed the peak (Figure 1C arrow) using SDS-PAGE and found the presence of both ColE1-T and TolC. The unimodal shifted peak observed with ColE1-T:TolC indicates that there is a single species of fully bound TolC. When resolved by cryoEM, addition of ColE1-T to TolC breaks the three-fold channel symmetry and the additional protein is observed running all the way through the TolC barrel as a single-pass, all- $\alpha$-helical chain spanning more than $130 \AA$ (Figure 1D). The maps refined to nominal global resolutions of $2.84 \AA$ and $3.09 \AA$ for 
A

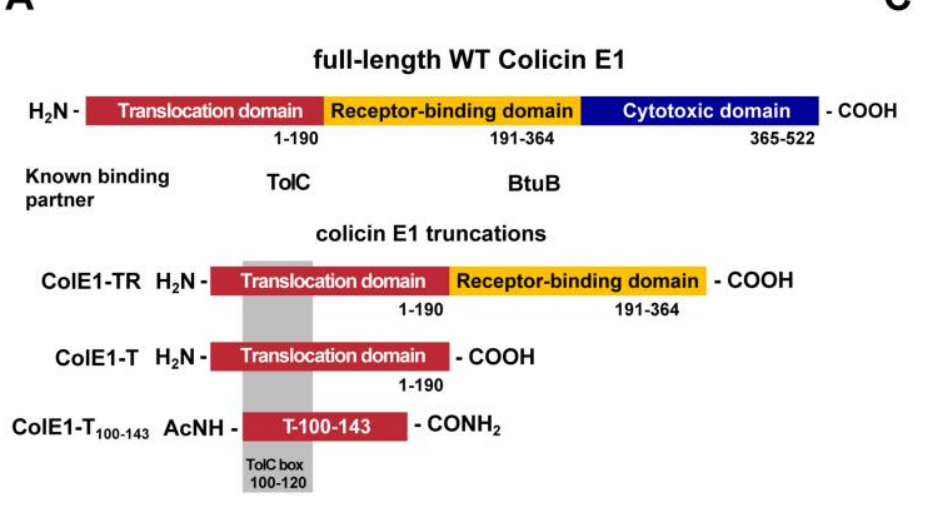

C
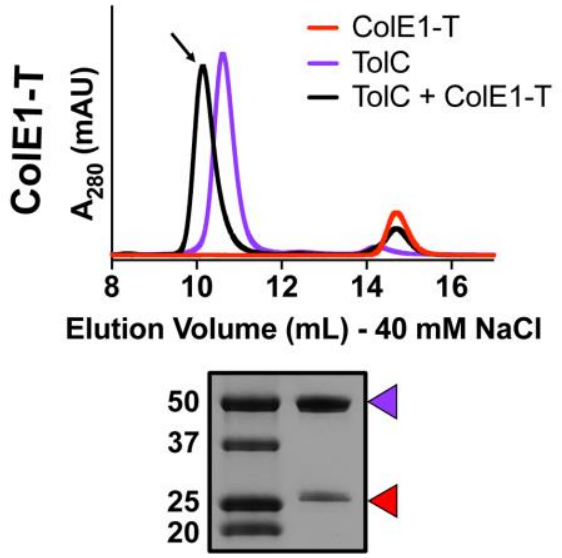

B
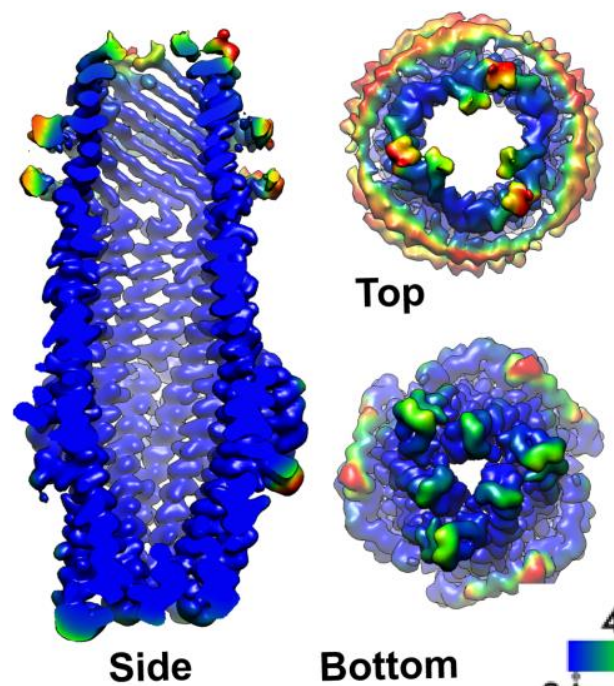

Bottom
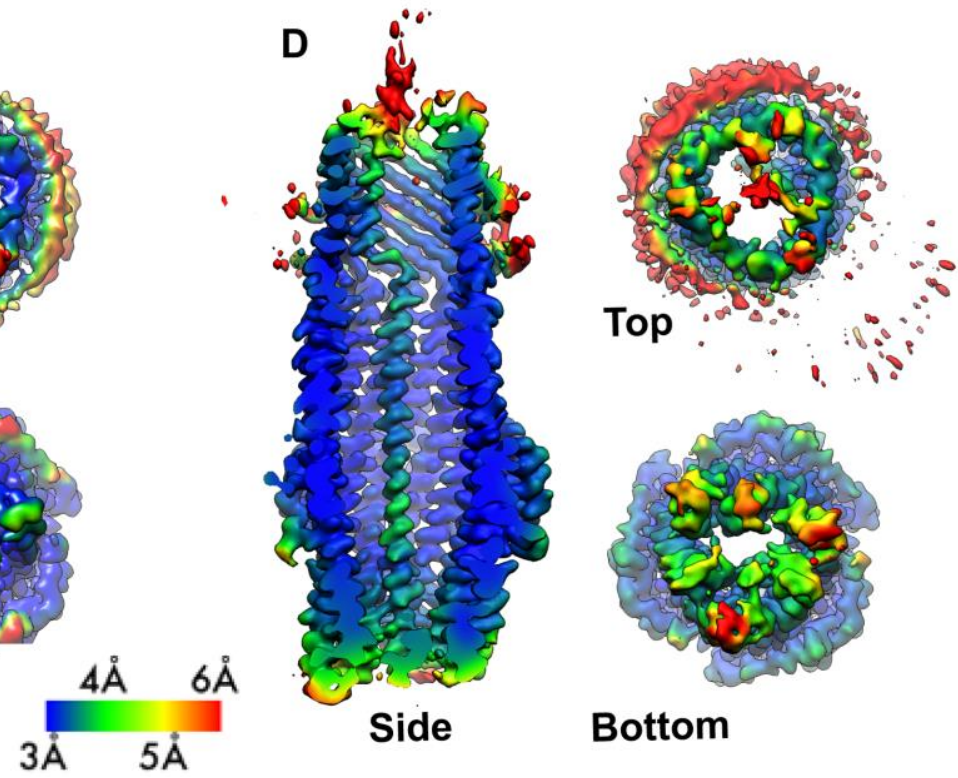

Bottom

Figure 1. Colicin E1-T binds within the lumen of TolC. (A) Architecture of full-length colicin E1 showing domains and their known binding partners. Three truncation constructs used in this study (B) CryoEM structure of TolC embedded in nanodiscs. Side, top, and bottom views are colored by local resolution, as computed in cryoSPARC from the final halfmaps. The side view is cropped to display the particle interior. (C) SEC chromatogram of ColE1-T (red line) and TolC (purple line). The arrow indicates the co-elution (black line) fractions (eluted in buffer containing $40 \mathrm{mM} \mathrm{NaCl}$ ) that were analyzed by SDS-PAGE. On the SDS-PAGE gel (bottom), red arrows indicate the presence of the colicin E1 construct that has co-eluted with TolC. SEC chromatogram of TolC purification (Figure 1 supplemental figure 1) and co-elution with $200 \mathrm{mM} \mathrm{NaCl}$ (Figure1 supplemental figure 2). (D) The CryoEM structure of ColE1-T bound to TolC and colored by local resolution as in (B). Cryo-EM data collection, refinement, and validation statistics in Figure 1, source data 1. 
space (Zakharov et al., 2016, Housden et al., 2013, Housden et al., 2018). The ColE1 chain binds

172 TolC asymmetrically, primarily contacting only one of the three TolC chains. 176 force.

The ColE1 T domain inserts into TolC with its amino terminus pointing inwards through the periplasmic iris, and 2D class-averages show that the carboxy terminus of the helix continues and projects out into the extracellular space (Figure 2A). 85 of the 190 ColE1-T residues could be modeled to this density (residues 46-131) (Figure 2B). No such regular protrusion was seen for the glycine-rich ColE1 amino-terminus, which we expect to be disordered in the periplasmic Compared to the unbound state, asymmetric ColE1 binding dilates the periplasmic TolC aperture (Figure 2C) and, makes only minor adjustments to the loops on the extracellular aperture (Figure 2D) so that they can accommodate ColE1 in the absence of any other proteins or motive

In solution, unbound colicin E1 is a two-helix hairpin as indicated by far UV circular dichroism (CD) (Zakharov et al., 2016). A structural model built using AlphaFold (Jumper et al., 2021) also predicts the closed hinge conformation with proline 110 at the apex (Figure 2E top). However, our cryoEM reconstruction of colicin E1 in complex with TolC shows that ColE1-T hinges open at the TolC box to an extended conformation upon binding to TolC (Figure $2 \mathrm{~B}$, and $2 \mathrm{E}$ bottom). The ColE1-T helices have a short kink in the middle around proline 110, precisely at the proposed turn location in the hairpin model (Figure 2B inset). This area is also the center of the TolC box (Figure 1A) that has been known to be critical for the ColE1:TolC interaction (Jakes, 2017, Zakharov et al., 2016). 


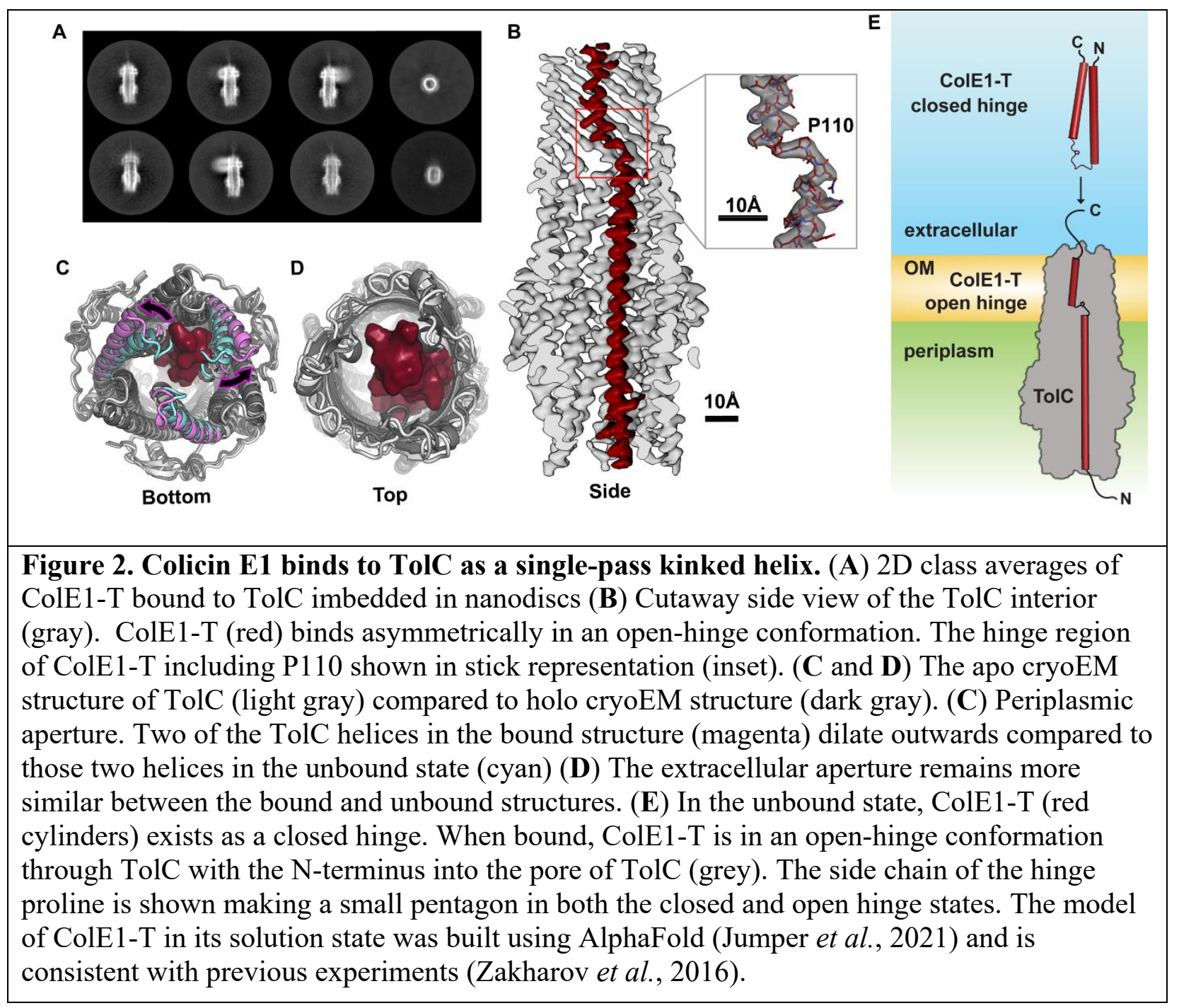




\section{Colicin E1 makes residue-specific interactions with TolC}

TolC forms a large rigid conduit in the outer membrane and periplasm (Figure 3A) with a water filled lumen. The hydrophilic nature of the channel (Dhar et al., 2021) coupled with the inflexibility of the outer-membrane-embedded barrel largely precludes the formation of any hydrophobic interfaces that are typically the basis of protein-protein interfaces involving helical peptides (Eisenberg et al., 1982). Yet, we did observe specific polar contacts between colicin E1's TolC box and the TolC barrel.

To obtain the most accurate atomic model for interpretation of atomic interactions between peptides, we utilized map-restrained molecular dynamics in model refinement (Wu et al., 2013). The refined model had improved chemical plausibility (for instance, the MolProbity (Williams et al., 2018) score improved from 1.25 to 0.50 ) and polar contacts were more easily identified. Because this refinement improved the concordance between the map and model, we conclude that the use of cryoEM density as a restraint was successful in preventing overfitting. Specifically, the EMRinger (Barad et al., 2015) score improved from 2.77 to 3.81 and the mapmodel FSC $=0.5$ improved from $3.44 \AA$ to $3.37 \AA$.

The atomic model showed significant polar interactions form between colicin E1 and TolC at the apertures and around the TolC box. The acidic patch of colicin E1 near the extracellular aperture contains two arginine residues (R129 and R131) that form hydrogen bond networks with one TolC chain each (Figure 3B). In addition, colicin E1 residues T113, A116, N119, N120 (all part of the TolC box) establish polar interactions with TolC residues G67, D69, S90 and K305 on one TolC chain (Figure 3C, light red and light blue) while colicin H117 interacts with TolC F310 on a neighboring TolC chain (Figure 3C, light red and light gold). These contacts are strong enough that even when the proline hinge of the TolC-box is mutated (P110A) ColE1-T still binds TolC (Figure 3, supplementary figure 2). 


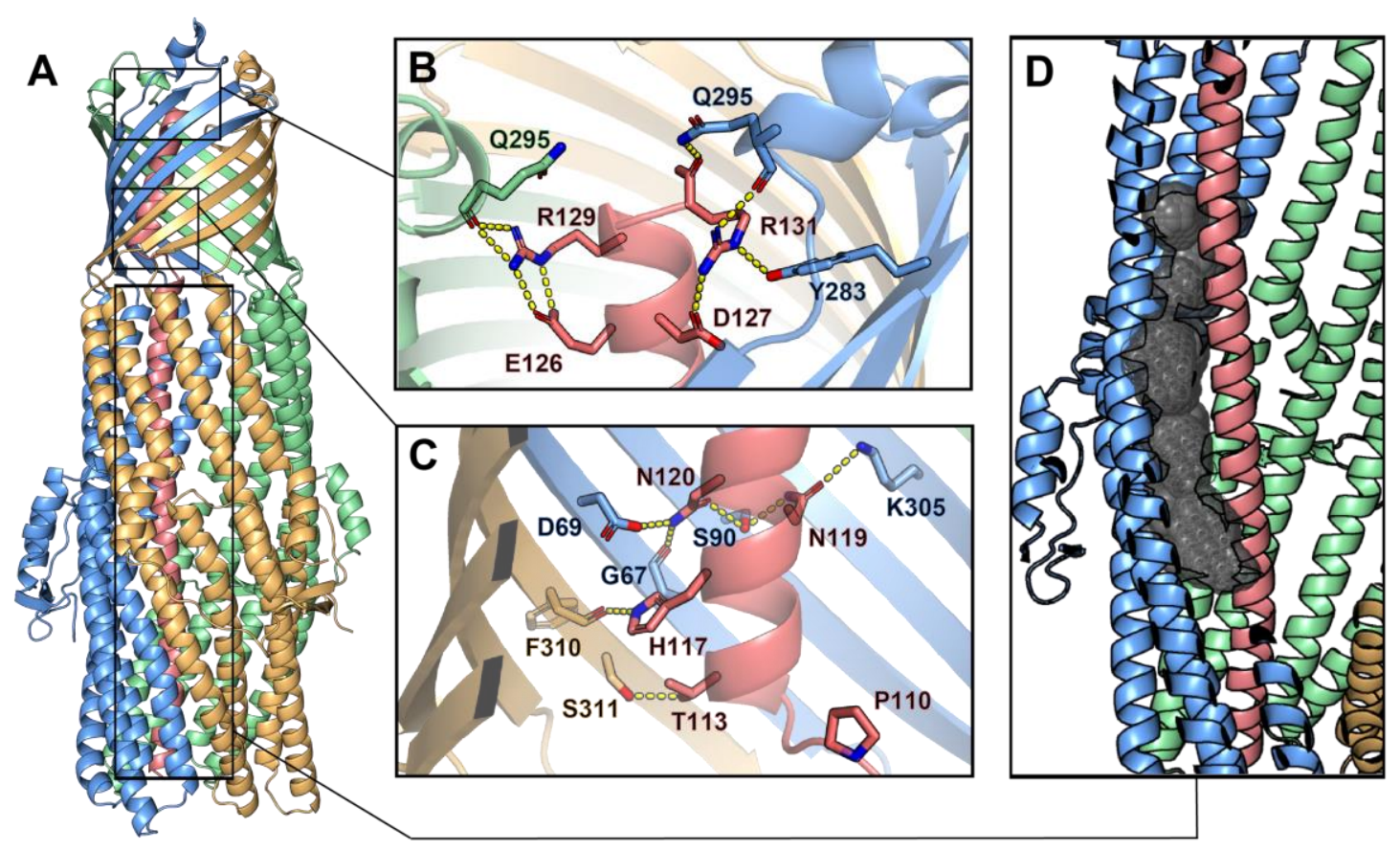

Figure 3. Inter-chain contacts between ColE1 T and TolC. (A) Molecular dynamics simulation refined structure of the ColE1-T:TolC complex colored by chain (ColE1-T: light red, TolC: light blue, light green, light gold) (B-C) Polar interaction network of ColE1-T and TolC on the $\mathrm{C}$-terminal side of the proline kink in the $\beta$-barrel region. Locations of previous mutagenesis data near the TolC box (figure 3 supplemental figure 1 and figure $3-$ video 1). (B) Near the extracellular opening of TolC and (C) in the TolC barrel near the transition to the TolC periplasmic helical region. Residues involved in forming polar interactions and proline 110 are shown in sticks. These interactions support binding such that even when the hinge proline is mutated to alanine (P110A) binding is maintained (Figure 3 supplemental figure 2). (D) ColE1-T spanning the TolC helical barrel does not make full contact with the side of the TolC barrel. The cavity (black spheres) between colicin (light red) and one chain of TolC (light blue) was detected using GHECOM (Kawabata, 2010, 2019, Kawabata \& Go, 2007).

By contrast with the $\beta$-barrel of TolC, in the interior of the periplasmic helical barrel of

217 TolC, colicin E1 does not make full contact with the $\alpha$-helical barrel interior and there is a gap

218 between the two proteins (Figure 3D, Figure 3-video 1). This is in agreement with previous

219 studies that showed a colicin truncation (residues 1-100), that ends just before the beginning of

220 the TolC box, does not bind to TolC (Zakharov et al., 2016) or prevent cytotoxicity of full-length

221 colicin in cells (Jakes, 2017) as this region of the colicin does not make strong interactions with

222 TolC (Figure 3D). 
Moreover, this structure is consistent with reports that mutations at TolC sites G65 or

S279 abrogate ColE1 activity (Masi et al., 2007) as these residues are contact sites between TolC and ColE1 (Figure 3 figure, supplemental figure 1 and Figure 3- video 1). Conversely, the absence of any effect of mutating colicin R103 and R108 (Jakes, 2017), is consistent with interaction those residues have with the solvent in the barrel rather than with TolC. The newly solved structure, in combination with previous work (Jakes, 2017, Zakharov et al., 2016), indicates the specificity of colicin E1 binding to TolC is encoded in the portion that binds to TolC $\beta$-barrel.

\section{Membrane localization of ColE1 truncations}

Since we find the ColE1 T and TR domains bind to TolC in vitro, we investigated if colicin E1 fragments bind to the native TolC in E. coli cells. Though ColE1 C domain activitydepolarizing the inner membrane-requires the $\mathrm{C}$ domain to pass through the outer membrane (Brunden et al., 1984), it remains unclear if the ColE1-TR domains translocate as well. We first determined that TolC binds to the TR domain in vitro similarly to TolC binding the T domain. ColE1-TR includes residues 1-364 (ColE1-TR) contains the N-terminal portion that binds to TolC and the C-terminal portion that binds to BtuB. ColE1-TR aggregates at the salt concentration (40 $\mathrm{mM}$ ) used for the co-elution experiment used for ColE1-T so we increased the $\mathrm{NaCl}$ concentration to $200 \mathrm{mM}$. ColE1-T still binds to TolC at $200 \mathrm{mM} \mathrm{NaCl}$ although to a much lesser degree (Figure 1 supplemental figure 1). Unlike ColE1-T (Figure 1C), ColE1-TR shows a bimodal distribution indicative of a mixed population of bound and unbound TolC (Figure 4A).

We assessed whether ColE1-T and ColE1-TR translocate into the cell or localize on the cell surface using an extracellular protease digestion assay (Besingi \& Clark, 2015). Colicin fragment stalling on the outer membrane surface would render the colicin fragments susceptible to digestion when trypsin is in the extracellular environment. Conversely, if the colicin fragment 
translocates into the intracellular environment, the outer membrane will shield the protein from digestion by trypsin.

251 Colicin fragment localization was probed through western blot analysis of specific colicin 252 fragments with C-terminal polyhistidine-tags. Cells were incubated with these colicin fragments 253 and subsequently exposed to two different concentrations of trypsin. If the protein enters the cell, 254 the outer membrane shields it from trypsin digestion, and a corresponding band is present on the western blot. Periplasmic protein SurA was used as a membrane integrity control. SurA digestion indicates that the periplasm was accessible to trypsin and a SurA band indicates that the periplasm was shielded from trypsin. As a trypsin activity control, half of the cell sample was lysed (lysed cell condition) exposing all cellular compartments to trypsin.

When probing for the C-terminal epitope by immunoblotting, there was no detectible ColE1-T binding (Figure 4B, left), though there was detectible binding of ColE1-TR (Figure 4B, right). Moreover, we found that if ColE1-TR is incubated with cells and subsequently treated with two different trypsin concentrations, the ColE1-TR band disappeared, indicating that the colicin

263 E1 fragment was localized to the outer membrane surface (Figure 4B, right). The periplasmic control SurA was not degraded at any trypsin concentration unless the cells were lysed before trypsin digestion (Besingi \& Clark, 2015).

We further probed the interaction between the cell membrane and surface-localized ColE1-TR with single-molecule fluorescence microscopy using the fluorescent dye Cy3 (Tuson \& Biteen, 2015, Al-Husini et al., 2020). When ColE1-TR-Cy3 was added to the extracellular environment of WT BW25113 E. coli (containing TolC), distinct puncta (Figure 4C, right) formed on $94 \%$ of the cells (number of cells, $n=111$ ) (Figure 4D); cells with observed puncta most often featured a single punctum, though a small fraction had 2 puncta. On average, the WT cells had 1.2 puncta. In a $\Delta t o l C$ strain, puncta were observed on only $18 \%$ of cells $(\mathrm{n}=99)$ (Figure 4C, right; Figure 4D); on average, the $\Delta t o l C$ cells had 0.2 puncta. As a $\Delta b t u B$ control 
274 strain, we used BL21 (DE3) which is known to have a premature stop codon at BtuB residue 58 275 (Studier et al., 2009). Puncta were observed on only 3\% of cells lacking BtuB ( $\mathrm{n}=105)$ (Figure

$2764 \mathrm{D}$ and Figure 4 supplemental figure 2A); on average, the $\Delta b t u B$ cells had 0 puncta.

The bright ColE1-TR-Cy3 puncta photobleach over time as they are observed in the fluorescence microscope, ultimately leaving a single molecule fluorescing before complete photobleaching. We therefore divided the brightness of each punctum by the brightness of that single molecule to estimate the number of molecules in each cluster (Bakshi et al., 2012). In WT and $\Delta$ tolC cells that featured puncta, ColE1-TR form puncta consistent with $\sim 20$ molecules; we never detect an isolated single ColE1-TR molecule on either WT or $\triangle$ tolC cells before photobleaching. The observed size and number of molecules agree with previous studies of BtuB clusters (Rassam et al., 2015, Chavent et al., 2018) and the punctum locations within the cell were variable. To rule out punctum formation as an artifact of Cy3 conjugation, we found ColE1-TRGFP displayed the same cluster formation as ColE1-TR-Cy3 (Figure 4 supplemental figure 2B).

Because we usually only see one punctum per cell, we anticipate that some BtuB and TolC may remain unbound because of the geometric constraints of punctum formation. ColE1 must engage both TolC and BtuB simultaneously. Therefore, both receptors must be in close proximity, similar to the $\sim 50 \AA$ proximity of BtuB to OmpF when both bound to a another type A colicin, ColE9 (Francis et al., 2021). Given the two-dimensional surface of a membrane there are restrictions to how many proteins can be within $50 \AA$ of any other protein. For the clusters we see to occur, when BtuB clusters together in groups of approximately 20 (Figure 4) TolC must also cluster in groups of approximately 20 , ultimately requiring approximately 40 proteins in a relatively small area. The competition between the clustering of BtuB and the requirement for proximity of BtuB and TolC for binding could lower the number of BtuB/TolC sites available for the $\mathrm{T}$ and $\mathrm{R}$ domains of colicin $\mathrm{E} 1$ due to the relative TolC and BtuB geometries needed for ColE1 binding. 
A

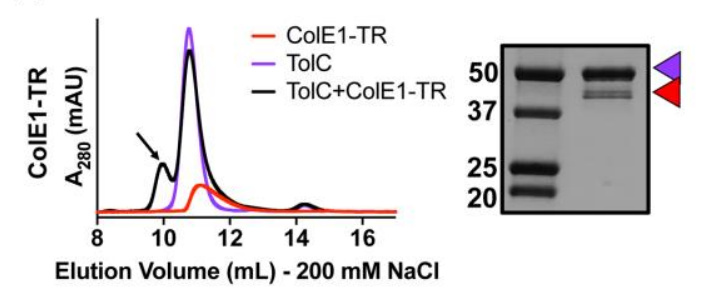

C

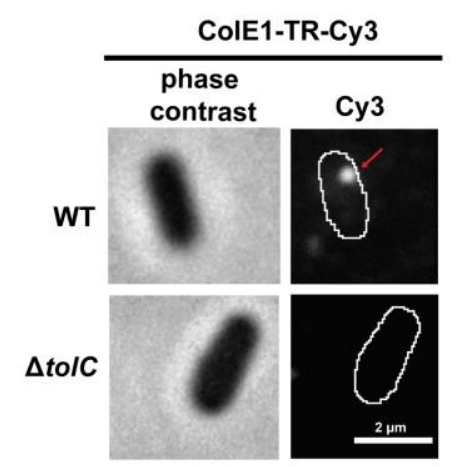

B

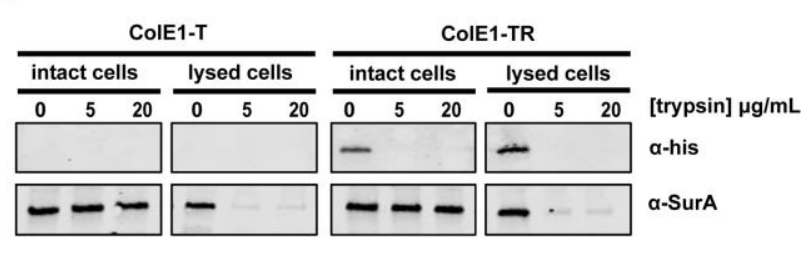

D

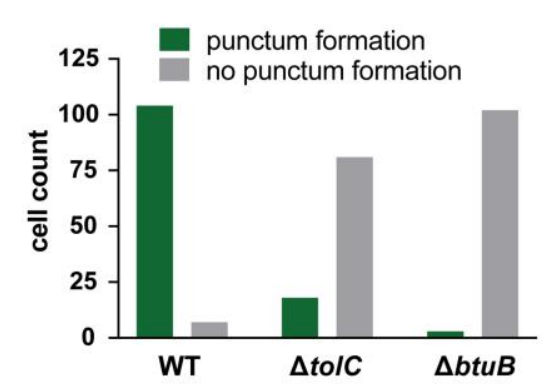

Figure 4. Colicin E1-TR localizes on the outside of the cell. (A) SEC chromatogram of ColE1-TR (red line) and TolC (purple line). The arrow indicates the co-elution (black line) fractions that were analyzed by SDS-PAGE. On the SDS-PAGE gels (right), red arrow indicates the presence of colicin E1 construct that has co-eluted with TolC (purple arrow). (B) Extracellular protease digestion assay with two colicin E1 truncation constructs incubated with $E$. coli, each construct is labeled with a C-terminal His-Tag. Periplasmic SurA was used as a membrane integrity control. After incubation with colicin fragments, cells were left intact or lysed with lysozyme. Proteins degraded by trypsin in intact cells are not protected by the outer membrane. (C) Fluorescence image (right) of ColE1-TR-Cy3 overlaid on outlines of living $E$. coli cells from phase-contrast microscopy (left) for WT and $\triangle$ tolC. Red arrow points to a punctum. A larger variety of images is also available (Figure 4 supplement 1). Similar localization was seen for ColE1-TR-GFP (Figure 4 supplemental figure 2) demonstrating that the effect was not caused by the fluorophore, and ColE1-TR $\mathrm{T}_{\Delta 1-}$ 40-Cy3 (Figure 4 supplemental figure 3, Figure 4, source data 1) demonstrating that the effect is not TolA dependent. The puncta were stable and were found to remain intact for more than five minutes. The punctum shown here remained intact for at least 30 seconds (Figure 4 - video 1). (D) Cell counts where ColE1-TR-Cy3 punctum formation was observed for WT, $\Delta t o l C$, and $\Delta b t u B$. Number of cells observed, $n=111,91,105$,

Fluorescently labeled pyocins, the colicin analog in Pseudomonas, have previously been

used to detect translocation across the outer membrane of Pseudomonas aeruginosa (White et al.,

2017). Here we use an analogous experiment with fluorescently labeled ColE1 to determine

cellular localization in E. coli. In time courses, bound ColE1-TR puncta remained immobile for >

5 minutes (Figure 4 - video 1 corresponds to 30 seconds of data used to attain the WT image in

Figure 4C). This is consistent with continued association of ColE1-TR with membrane-embedded 
305 BtuB, which has limited mobility (Kleanthous et al., 2015). This result indicates that ColE1-TR 306 does not fully translocate (White et al., 2017), because if ColE1-TR entered the periplasmic space 307 it would freely diffuse on these timescales.

Colicin constructs lacking the R domain (ColE1-T-Cy3) showed no detectable binding 309 either to WT or $\Delta$ tolC cells (Figure 4 supplemental figure 2C), indicating that the TolC-ColE1-T 310 interaction is weaker than the BtuB-ColE1-TR interaction, likely due to the absence of the R 311 domain-BtuB interaction.

\section{ColE1-TR inhibits efflux and makes $E$. coli more susceptible to antibiotics}

Since the colicin truncations are stalled on their respective OMP receptors, we investigated if this stalling could disrupt native TolC efflux. Real-time efflux inhibition by colicin E1 fragments was assessed using a live-cell assay with N-(2-naphthyl)-1-naphthylamine (NNN)dye, which passively diffuses into cells and is effluxed by the AcrAB-TolC efflux pump. NNN 
fluoresces when it is localized in the hydrophobic interior of one of the membranes (Bohnert $e t$ al., 2011) thereby acting as a reporter for the relative rates of diffusion and efflux. NNN efflux

332 can be turned off by the protonophore CCCP, which neutralizes the proton motive force and

allows NNN to accumulate within the cell. Active efflux can then be monitored by the

fluorescence decay once proton motive force is reenergized by glucose addition (Bohnert et al., 2010, Seeger et al., 2008, Bohnert et al., 2011, Iyer et al., 2015, Misra et al., 2015).

We assessed the ability of colicin E1 truncations to plug TolC by monitoring real-time NNN efflux. Cells exposed to ColE1- $\mathrm{T}_{100-143}$ did not show lower real-time efflux than untreated cells (Figure 5A). This observation is notable in light of the fact that, in previous conductance studies, similar peptides were shown to bind TolC (Zakharov et al., 2016, Jakes, 2017) and to occlude the channel (Zakharov et al., 2016, Zakharov et al., 2004).

Cells exposed to high concentrations of ColE1-T showed less decay in final fluorescence than untreated cells, indicating that ColE1-T partially inhibits efflux (Figure 5B). Finally, exposure to high concentrations of ColE1-TR produced no decrease in fluorescence, showing full inhibition of efflux (Figure 5C).

Because ColE1-TR completely inhibits NNN efflux, we evaluated the capacity of ColE1TR to potentiate antibiotics representing three different classes that are known TolC substrates: ciprofloxacin, erythromycin, and benzalkonium chloride (from fluoroquinolones, macrolides, and quaternary ammonium compounds, respectively). An effective TolC plug will reduce the concentration required to inhibit growth as antibiotics remain trapped within the cell. WT E. coli cells that were exposed to $100 \mu \mathrm{M}$ ColE1-TR with each of these antibiotics showed significantly lower minimum inhibitory concentrations (MICs), the lowest concentration of antibiotic that inhibits visible growth, than cells exposed to the antibiotics alone (Figure 5D): exposure to 100 $\mu \mathrm{M}$ ColE1-TR made WT E. coli $\sim 2$ to 7 -fold more susceptible to these antibiotics (Figure 5, 
354 source data 1). Exposure of WT cells to $10 \mu \mathrm{M}$ ColE1-TR was sufficient to potentiate 355 erythromycin. 

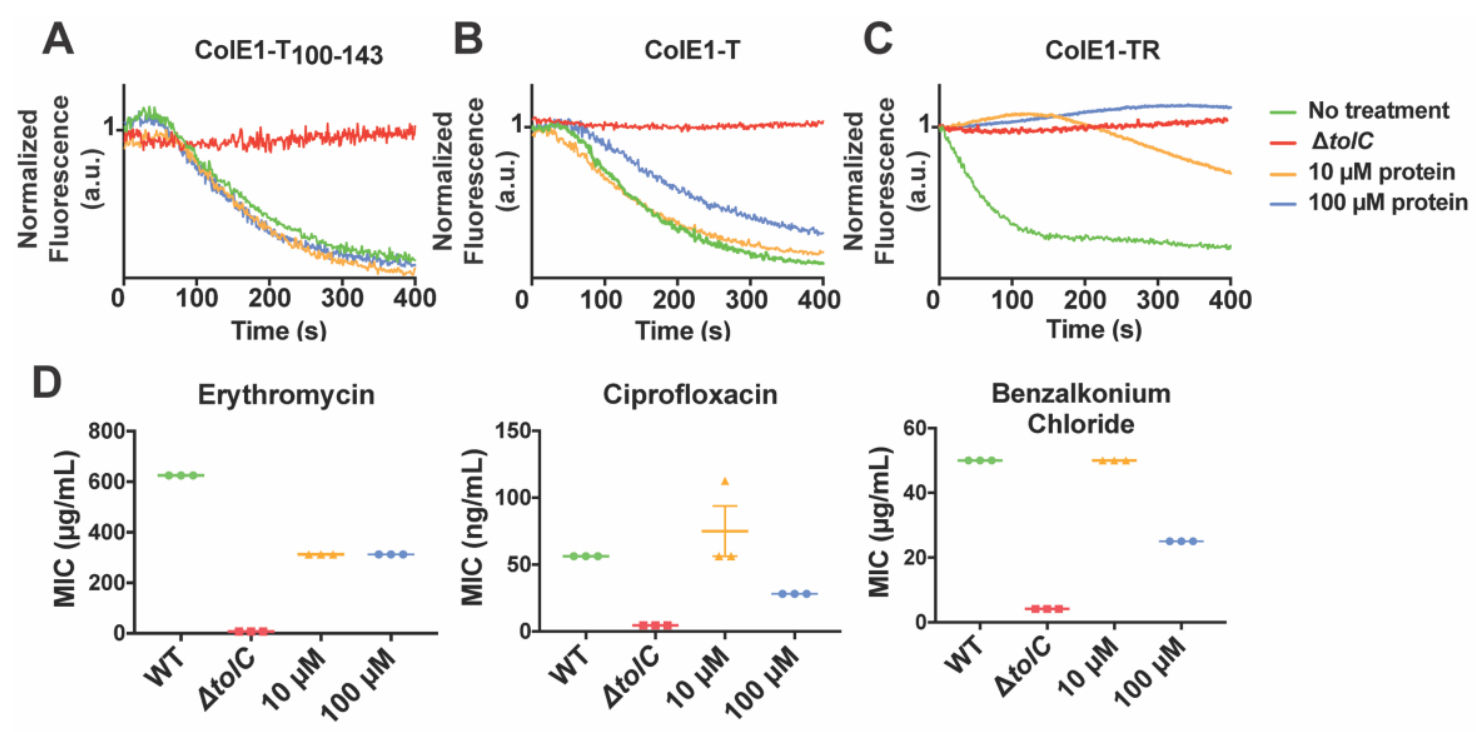

Figure 5. Colicin E1 fragments inhibit efflux and potentiate antibiotics.

(A-C) Effect of colicin E1 fragments on efflux: WT with no protein (green), $\Delta t o l C$ (red), $\mathrm{WT}+10 \mu \mathrm{M}$ (orange), WT $+100 \mu \mathrm{M}$ protein (blue). (A) ColE1-T $100-143$, (B) ColE1-T, (C) ColE1-TR. All efflux experiments used $10 \mu \mathrm{M}$ of fluorescent reporter dye NNN. (D) Antibiotic susceptibilities in the absence (green) and presence of ColE1-TR added to WT at $10 \mu \mathrm{M}$ (orange) and $100 \mu \mathrm{M}$ (blue). MICs for $\Delta t o l C$ (red) are included as a reference. Data shown from 3 biological replicates reported as the mean and individual data points. Bars indicate standard error of the mean. Data values shown in Figure 5, source data 1.

TolA is normally involved in outer membrane invagination during cell division (Gerding et al., 2007). Like Colicin E1, Colicin E9 contains an intrinsically unstructured N-terminus that engages the Tol-Pal system once it penetrates into the periplasm (Loftus et al., 2006).

Engagement of the Tol-Pal system by the N-terminal region ofcolicin E9 disrupts native Tol-Pal system function leading to outer membrane defects (Rassam et al., 2018). Outer membrane defects may lead to enhanced antibiotic susceptibility. To rule out outer membrane-mediated defects as the main contributor to the reduction in MICs seen in Figure 5, we tested the effect of using a truncation of ColE1-TR $\left(\right.$ ColE1-TR $\left.{ }_{\Delta 1-40}\right)$ lacking the $\mathrm{N}$-terminal 40 residues including the TolA box. The MIC of ColE1-TR and ColE1-TR ${ }_{\Delta 1-40}$ are identical (Figure5 supplemental figure 1) indicating that ColE1-TR engagement of TolA does not contribute to the observed antibiotic susceptibility. 


\section{Discussion}

There are two conflicting models of ColE1 translocation: 1) the total thread model in which the entire colicin unfolds and threads through TolC (Cramer et al., 2018, Jakes, 2017, Francis et al., 2021) and 2) the pillar model, in which colicin E1 inserts into TolC as a helical hairpin, facilitating LPS-mediated, self-translocation of the colicin cytotoxic domain (Zakharov et al., 2016). Our data support aspects of both models. The belief in a closed hinge conformation of bound ColE1 prompted two arguments against the total thread mechanism: 1) the ColE1-T closed hinge conformation is too wide to fit through TolC and 2) both ends would face the extracellular milieu (Zakharov et al., 2016, Cramer et al., 2018). The ColE1-T hinge opening in the bound state — which was unanticipated by the pillar model— resolves these objections: colicin threads into the TolC barrel with the amino terminus pointing into the periplasm, as the total thread model hypothesized. Our trypsin digests show ColE1-TR stalls at the outer membrane of $E$. coli cells and the single-molecule fluorescence images confirm that ColE1-TR remains clustered and does not diffuse as would be expected if entering the periplasm or cytoplasm. These puncta form regardless of the presence or absence of the TolA binding region on ColE1-TR.

Though we cannot exclude the possibility that ColE1 $\mathrm{T}$ and $\mathrm{R}$ domain translocation depends on the $\mathrm{C}$ domain, we observe—as hypothesized by the pillar model—-that colicin sits inside TolC but does not translocate. These results are in agreement with early studies of poreforming colicins in which trypsin added to the extracellular environment can reverse colicin activity (Plate \& Luria, 1972, Dankert et al., 1980, Dankert et al., 1982, Benedetti et al., 1992, Duche, 2007). We anticipate that the stable ColE-TR:TolC complex forms regardless of the presence of the $\mathrm{C}$ domain.

If the $\mathrm{C}$ domain does translocate while the $\mathrm{T}$ and $\mathrm{R}$ domains remain bound, we anticipate that the requirement for TolA is due to its role in membrane maintenance. Recruitment of the Tol- 
Pal system by a different group A colicin (ColE9) causes membrane defects similar to those in a

$\Delta$ tolA strain (Rassam et al., 2018). Therefore, in the pillar model, colicin binding of TolA may

\section{9}

facilitate $\mathrm{C}$ domain insertion through the defective membrane, whereas in the total thread model

binding TolA transduces the proton motive force to unfold ColE1 and pull it into the periplasm

(Figure 6, supplemental figure 1). Because we did not use the toxic $\mathrm{C}$ domain, our data cannot

clarify what occurs after the $\mathrm{T}$ and $\mathrm{R}$ domain of full length colicin bind the outer membrane

proteins. Although our studies find that ColE1-TR remains on the surface of the bacteria, it is

certain that the $\mathrm{C}$ domain, when present, translocates across the membrane into the target bacteria

to kill the cell. Our data does not exclude the possibility that the translocation of the $\mathrm{T}$ and $\mathrm{R}$

domains occurs along with the $\mathrm{C}$ domain in full length colicin, nor does it support $\mathrm{T}$ or $\mathrm{R}$ domain

translocation. Thus, though our studies give a more complete view of the early stages of ColE1

binding the surface of its target, we cannot determine if insertion of the $\mathrm{C}$ domain occurs via the total thread model or via a modified pillar model.

Shortly after this work was made available, a similar structure demonstrated that KlebC,

an rRNAse bacteriocin from Klebsiella pneumoniae, binds TolC from Klebsiella

quasipneumoniae with a binding mode similar to that of ColE1 binding TolC.(Housden et al.,

Figure 6. Model of colE1-TR inhibition of efflux. The R domain of ColE1-TR (orange) binds to BtuB (cyan) with high affinity and anchors colE1-TR to the surface of the cell. The T domain (red) then inserts into TolC (lavender), plugging the channel and blocking exit of antibiotics (spheres). We only see this interaction when $\sim 20$ colE1-TR bind in the same cluster though for simplicity only 12 BtuB and 12 TolC proteins are pictured here. (A) Before binding, antibiotics are effluxed (B) After binding the colicin E1 fragments prevent some antibiotic efflux.

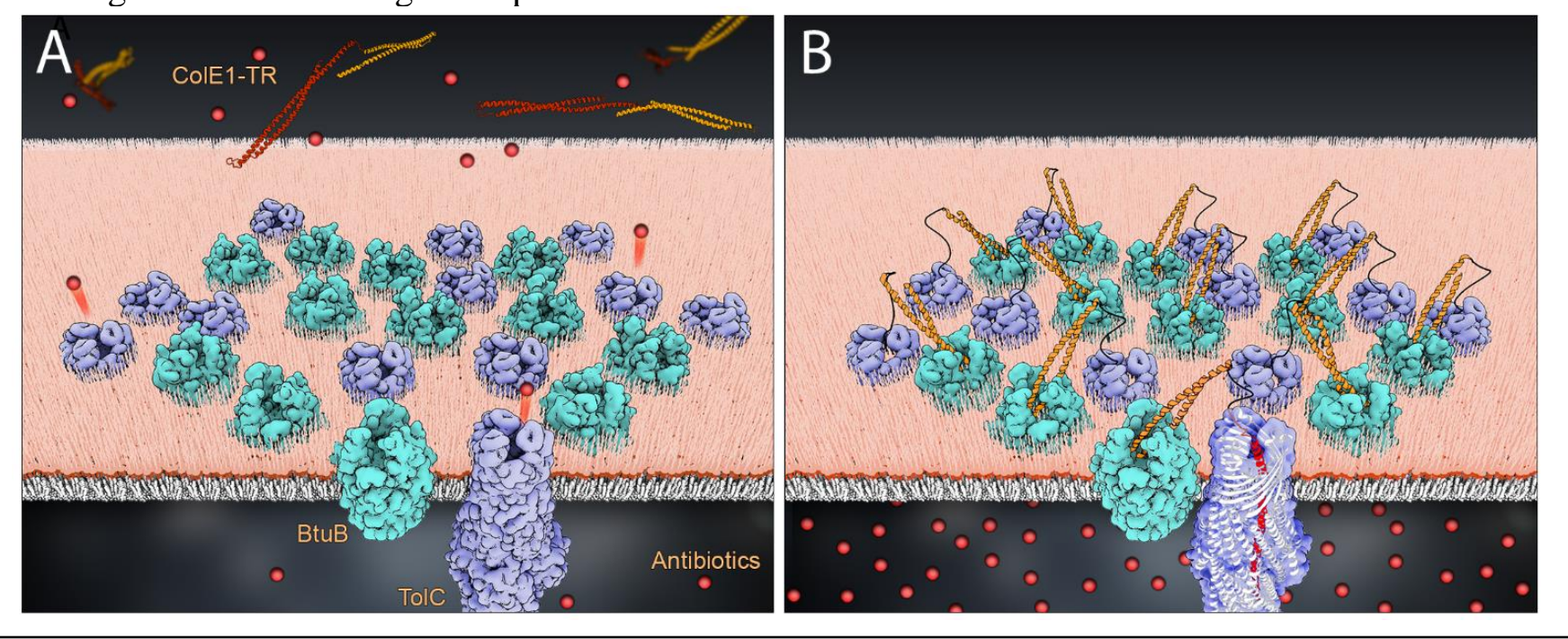


413 2021) KlebC is closed in the unbound state as confirmed by the high-resolution x-ray crystal 414 structure, which supports our proposed conformation (Figure 2E), and opens into a single-pass, 415 kinked-helix to bind to TolC. Taken together, these findings suggest a conserved mechanism for 416 bacteriocins that hijack trimeric efflux pump proteins.

\section{Materials and Methods}

\section{Key Resources Table}




\begin{tabular}{|c|c|c|c|c|}
\hline $\begin{array}{l}\text { Reagent } \\
\text { type } \\
\text { (species) or } \\
\text { resource }\end{array}$ & Designation & $\begin{array}{l}\text { Source or } \\
\text { reference }\end{array}$ & Identifiers & $\begin{array}{l}\text { Additional } \\
\text { information }\end{array}$ \\
\hline Antibody & $\begin{array}{l}\text { THE HisTag mAb } \\
\text { Mouse (mouse } \\
\text { monoclonal) }\end{array}$ & GenScript & $\begin{array}{l}\text { Cat\#A00186-100; } \\
\text { RRID:19J001957 }\end{array}$ & $(1: 2,000)$ \\
\hline Antibody & $\begin{array}{l}\text { anti-SurA Rabbit } \\
\text { polyclonal (rabbit } \\
\text { polyclonal) }\end{array}$ & Cusabio & $\begin{array}{l}\text { Cat\#CSB- } \\
\text { PA359693HA01EN } \\
\text { V; RRID:E0109A }\end{array}$ & $(1: 2,500)$ \\
\hline Antibody & $\begin{array}{l}\text { IRDye } 800 \mathrm{CW} \text { goat anti- } \\
\text { mouse IgG secondary } \\
\text { (goat polyclonal) }\end{array}$ & LI-COR & $\begin{array}{l}\text { Cat\#925-32210; } \\
\text { RRID:C81106-01 }\end{array}$ & $(1: 10,000)$ \\
\hline Antibody & $\begin{array}{l}\text { IRDye 800CW Donkey } \\
\text { anti-Rabbit (donkey } \\
\text { monoclonal) }\end{array}$ & LI-COR & $\begin{array}{l}\text { Cat\#925-32213; } \\
\text { RRID:C80829-10 }\end{array}$ & $(1: 10,000)$ \\
\hline $\begin{array}{l}\text { Cell line }(E . \\
\text { coli })\end{array}$ & BW25113 & $\begin{array}{l}\text { Coli Genetic } \\
\text { Stock } \\
\text { Center }\end{array}$ & Cat\#7636 & \\
\hline $\begin{array}{l}\text { Cell line }(E . \\
\text { coli })\end{array}$ & JW5503-1 & $\begin{array}{l}\text { Coli Genetic } \\
\text { Stock } \\
\text { Center }\end{array}$ & Cat\#11430 & \\
\hline $\begin{array}{l}\text { Cell line }(E . \\
\text { coli })\end{array}$ & BL21(DE3) & $\begin{array}{l}\text { Coli Genetic } \\
\text { Stock } \\
\text { Center }\end{array}$ & Cat\#12504 & \\
\hline $\begin{array}{l}\text { Recombinant } \\
\text { DNA reagent }\end{array}$ & pTrc99a-NoSS-TolC & This paper & Addgene: 155179 & $\begin{array}{l}\text { Addgene: } \\
155179 \\
\end{array}$ \\
\hline $\begin{array}{l}\text { Recombinant } \\
\text { DNA reagent }\end{array}$ & pET303-colE1-T & This paper & Addgene: 155180 & $\begin{array}{l}\text { Addgene: } \\
155180\end{array}$ \\
\hline $\begin{array}{l}\text { Recombinant } \\
\text { DNA reagent }\end{array}$ & pET303-colE1-TR & This paper & Addgene: 155181 & $\begin{array}{l}\text { Addgene: } \\
155181\end{array}$ \\
\hline $\begin{array}{l}\text { Recombinant } \\
\text { DNA reagent }\end{array}$ & pET303-colE1-T-E192C & This paper & Addgene: 180233 & $\begin{array}{l}\text { Addgene: } \\
180233\end{array}$ \\
\hline $\begin{array}{l}\text { Recombinant } \\
\text { DNA reagent }\end{array}$ & $\begin{array}{l}\text { pET303-colE1-TR- } \\
\text { E366C }\end{array}$ & This paper & Addgene: 180234 & $\begin{array}{l}\text { Addgene: } \\
180234 \\
\end{array}$ \\
\hline $\begin{array}{l}\text { Recombinant } \\
\text { DNA reagent }\end{array}$ & $\begin{array}{l}\text { pET303-colE1-TR- } \\
\text { E366C- } \Delta 1-40\end{array}$ & This paper & Addgene: 180235 & $\begin{array}{l}\text { Addgene: } \\
180235\end{array}$ \\
\hline $\begin{array}{l}\text { Recombinant } \\
\text { DNA reagent }\end{array}$ & pET303-colE1-TR- $\Delta 1-40$ & This paper & Addgene: 180236 & $\begin{array}{l}\text { Addgene: } \\
180236\end{array}$ \\
\hline $\begin{array}{l}\text { Recombinant } \\
\text { DNA reagent }\end{array}$ & pET303-colE1-T-P110A & This paper & Addgene: 180237 & $\begin{array}{l}\text { Addgene: } \\
180237\end{array}$ \\
\hline $\begin{array}{l}\text { Recombinant } \\
\text { DNA reagent }\end{array}$ & MSP1D1 & Addgene & Addgene: 20061 & \\
\hline $\begin{array}{l}\text { Software, } \\
\text { Algorithm }\end{array}$ & cryoSPARC 2 v2.12 & $\begin{array}{l}\text { Punjani, } \\
\text { Rubinstein } \\
\text { et al. } 2017\end{array}$ & $\begin{array}{l}\text { https://cryosparc.co } \\
\text { m/ }\end{array}$ & \\
\hline
\end{tabular}




\begin{tabular}{|l|l|l|l|l|}
$\begin{array}{l}\text { Software, } \\
\text { Algorithm }\end{array}$ & phenix v1.14-3260 & $\begin{array}{l}\text { Afonine, } \\
\text { Poon et al. } \\
2018\end{array}$ & $\begin{array}{l}\text { https://www.phenix- } \\
\text { online.org/ }\end{array}$ & \\
\hline $\begin{array}{l}\text { Software, } \\
\text { Algorithm }\end{array}$ & serialEM v3.7 & $\begin{array}{l}\text { Mastronarde } \\
2018\end{array}$ & $\begin{array}{l}\text { https://bio3d.colorad } \\
\text { o.edu/SerialEM/ }\end{array}$ & \\
\hline $\begin{array}{l}\text { Software, } \\
\text { Algorithm }\end{array}$ & coot v0.8.9.2-re 7766 & $\begin{array}{l}\text { Emsley, } \\
\text { Lohkamp et } \\
\text { al. 2010 }\end{array}$ & $\begin{array}{l}\text { https://www2.mrc- } \\
\text { lmb.cam.ac.uk/perso } \\
\text { nal/pemsley/coot/ }\end{array}$ & \\
\hline & ISOLDE v1.0b4.dev1 & $\begin{array}{l}\text { Croll 2018 } \\
\text { Software, } \\
\text { Algorithm }\end{array}$ & $\begin{array}{l}\text { https://isolde.cimr.ca } \\
\text { /doc/tools/ISOLDE. } \\
\text { html }\end{array}$ & \\
\hline $\begin{array}{l}\text { Software, } \\
\text { Algorithm }\end{array}$ & UCSF ChimeraX & $\begin{array}{l}\text { Goddard, } \\
\text { Huang et al. } \\
\text { 2018 }\end{array}$ & $\begin{array}{l}\text { https://www.cgl.ucsf } \\
\text {.edu/chimerax/ }\end{array}$ & \\
\hline $\begin{array}{l}\text { Software, } \\
\text { Algorithm }\end{array}$ & PyMOL & $\begin{array}{l}\text { Schrödinger } \\
\text { LLC }\end{array}$ & https://pymol.org/2/ & \\
\hline
\end{tabular}

E. coli strains. E. coli strains BW25113 and JW5503-1 were purchased from the Coli Genetic

Stock Center (CGSC). JW5503-1 is a tolC732(del)::kan from the parent strain BW25113.

BL21(DE3) were used for production of the colicin constructs and TolC. BL21(DE3) has a

premature stop codon at residue 58 of the $b t u B$ gene and therefore we used it as a $\triangle b t u B$ strain for microscopy.

Expression and Purification. The gene for ColE1-TR was synthesized as a gBlock (Integrated DNA Technologies) and cloned into pET303. Inverse PCR was used to delete the R domain and produce colicin E1-T. Colicin E1-TR-GFP was produced by inserting GFP at the C terminus of ColE1-TR. A gBlock (Integrated DNA Technologies) was synthesized for GFP containing complimentary flanking sequences to the plasmid with ColE1-TR and inserted with the omegaPCR technique. ColE1-TR $\mathrm{TR}_{\Delta 1-40}$ was produced by inverse PCR to delete residues corresponding to 1 through 40. All point mutants (ColE1-T-E192C, ColE1-TR-E366C, ColE1-TR-E366C ${ }_{\Delta 1-40}$, and ColE1-T-P110A) were generated by inverse PCR with mutagenic primers. 
Plasmids were transformed into E. coli BL21(DE3) cells and plated on LB + agar +100

$\mu \mathrm{g} / \mathrm{mL}$ carbenicillin. Single colonies were inoculated into $50 \mathrm{~mL}$ LB broth with $100 \mu \mathrm{g} / \mathrm{mL}$

carbenicillin and grown overnight at $37^{\circ} \mathrm{C}$ with shaking at 250 r.p.m. Proteins were produced by inoculating $1 \mathrm{~L}$ of TB supplemented with $0.4 \%$ glycerol, $10 \mathrm{mM} \mathrm{MgCl}_{2}$ and $100 \mu \mathrm{g} / \mathrm{mL}$

carbenicillin with $20 \mathrm{~mL}$ of the overnight culture. The culture was grown at $37^{\circ} \mathrm{C}$ to an OD600 of 2.0 and induced with $1 \mathrm{mM} \mathrm{IPTG}$. Expression cultures were then grown at $15{ }^{\circ} \mathrm{C}$ for 24 hours and harvested at 4,000 g for 30 minutes at $4{ }^{\circ} \mathrm{C}$. Cell pellets were resuspended at $3 \mathrm{~mL} / \mathrm{g}$ of cell pellet in lysis buffer (TBS, $5 \mathrm{mM} \mathrm{MgCl} 2,10 \mathrm{mM}$ imidazole, $1 \mathrm{mM}$ PMSF, $10 \mu \mathrm{g} / \mathrm{mL}$ DNase, 0.25 $\mathrm{mg} / \mathrm{mL}$ lysozyme) and lysed via sonication (2 minutes, $2 \mathrm{~s}$ on, $8 \mathrm{~s}$ off, $40 \%$ amplitude, QSonica Q500 with $12.7 \mathrm{~mm}$ probe) in an ice bath. Lysates were centrifuged at 4,000 $\mathrm{g}$ for 10 minutes to remove un-lysed cells and debris. The supernatant was centrifuged again at 50,400 $\mathrm{g}$ in a Beckman Coulter J2-21 for 1 hour at $4{ }^{\circ} \mathrm{C}$. Clarified lysates were applied to a $5 \mathrm{~mL}$ HisTrap FF column and purified using an ÄKTA FPLC system with a 20-column volume wash step with binding buffer (TBS, $25 \mathrm{mM}$ imidazole) and eluted using a linear gradient from 0-50\% elution buffer (TBS, $500 \mathrm{mM}$ imidazole) in 10 column volumes. Concentrated proteins were loaded onto a HiLoad Superdex 16/60 200 pg gel filtration column and eluted into phosphate buffered saline (PBS) $\mathrm{pH} 7.4$.

TolC production and purification was conducted in the same manner for preparation for cryoEM and for SEC. The gene for full-length TolC (a generous gift from R. Misra) was cloned into pTrc99a with the signal sequence deleted for expression into inclusion bodies. Plasmids were transformed into BL21(DE3) and plated on $\mathrm{LB}+$ agar $+100 \mu \mathrm{g} / \mathrm{mL}$ carbenicillin. A single colony was picked and grown in $\mathrm{LB}$ at $37{ }^{\circ} \mathrm{C}$ with shaking at 250 r.p.m. overnight. In the morning, $1 \mathrm{~L}$ of LB was inoculated with $20 \mathrm{~mL}$ of the overnight culture and grown at $37^{\circ} \mathrm{C}$ with shaking at 250 r.p.m. until the culture reached an OD600 of 0.6, at which point protein expression was induced with $1 \mathrm{mM}$ IPTG for an additional 4 hours. Cells were then harvested at 4,000g for 30 minutes at 4 
${ }^{\circ} \mathrm{C}$. Cell pellets were resuspended in $\mathrm{mL}$ of lysis buffer (TBS, $5 \mathrm{mM} \mathrm{MgCl}, 0.2 \mathrm{mg} / \mathrm{mL}$

lysozyme, $5 \mu \mathrm{g} / \mathrm{mL}$ DNase, $1 \mathrm{mM}$ PMSF) at $3 \mathrm{~mL} / 1 \mathrm{~g}$ of cell pellet and lysed via sonication (4

483 minutes, 2s on, 8s off, 40\% amplitude, QSonica Q500 with $12.7 \mathrm{~mm}$ probe) in an ice bath. Cell

484 lysates were centrifuged at $4,000 \mathrm{~g}$ for 30 minutes at $4{ }^{\circ} \mathrm{C}$ to harvest inclusion bodies. Inclusion

485 body pellets were resuspended in inclusion body wash buffer (20 mM Tris pH 8.0, 0.5 mM

EDTA, $1 \%$ Triton $\mathrm{X}-100$ ) and centrifuged at 4,000g for 30 minutes at $4{ }^{\circ} \mathrm{C}$. The inclusion body

wash was repeated two more times. A final wash was done in $20 \mathrm{mM}$ Tris $\mathrm{pH} 8.0$ and inclusion

bodies were stored at $-20^{\circ} \mathrm{C}$. Inclusion bodies were solubilized in $20 \mathrm{mM}$ Tris $\mathrm{pH}$ 8.0, $8 \mathrm{M}$ urea at $500 \mu \mathrm{M}$. N-octylpolyoxyethylene was added to $5 \mathrm{~mL}$ of solubilized TolC to a final concentration of $10 \%$ detergent and pipetted into a Slide-A-Lyzer G2 dialysis cassette with a 10,000 molecular weight cut off. Refolding was initiated by dialysis in $5 \mathrm{~L}$ of $20 \mathrm{mM}$ Tris $\mathrm{pH} 8.0,100 \mathrm{mM} \mathrm{NaCl}$ at $4{ }^{\circ} \mathrm{C}$ with stirring overnight. TolC was centrifuged at $15,200 \mathrm{~g}$ for 10 minutes at $4{ }^{\circ} \mathrm{C}$ to remove aggregates. The supernatant was filtered through a $0.22 \mu \mathrm{m}$ filter, concentrated to $2 \mathrm{~mL}$, applied onto a HiLoad 16/60 Superdex 200 pg column on an ÄKTA Pure FPLC system, and eluted with 1.5 column volumes in $20 \mathrm{mM}$ Tris $\mathrm{pH} 8.0,100 \mathrm{mM} \mathrm{NaCl}, 0.05 \%$ n-dodecyl- $\beta$-D-maltoside. TolC containing fractions were pooled and concentrated to $300 \mu \mathrm{M}$ in Amicon centrifugal filters with molecular weight cutoff of 30kDa.

Membrane scaffold protein MSP1D1. MSP1D1 in pET28a was purchased from Addgene and was expressed and purified as previously described(Hagn et al., 2018).

Peptide Synthesis. ColE1-T100-143 was synthesized using standard Fmoc chemistry with a CEM liberty blue microwave peptide synthesizer. The peptides were cleaved using a solution of 92.5:2.5:2.5:2.5 TFA:TIPS:H2O:DoDt and the crude peptides were purified using HPLC. Analytical HPLC traces were acquired using an Agilent 1100 quaternary pump and a Hamilton PRP-1 (polystyrene-divinylbenzene) reverse phase analytical column ( $7 \mu \mathrm{m}$ particle size, $4 \mathrm{~mm} \mathrm{x}$ 
$25 \mathrm{~cm}$ ) with UV detection at $215 \mathrm{~nm}$. The eluents were heated to $45^{\circ} \mathrm{C}$ to reduce separation of rotational isomers, and elution was achieved with gradients of water/ acetonitrile (90:10 to 0:100

Waters Micromass ZQ 4000 instrument with ESI+ ionization.

\section{Extracellular Protease Digestion.}

Growth, incubation with protein, and washing: $5 \mathrm{~mL}$ overnight cultures of E. coli

(BW25113) were grown in LB meda at $37^{\circ} \mathrm{C}$ with shaking (250 r.p.m.) overnight. A $50 \mathrm{~mL}$

culture of $\mathrm{LB}$ was inoculated with $1 \mathrm{~mL}$ of the overnight culture and grown at $37^{\circ} \mathrm{C}$ with shaking (250 r.p.m.) until an OD600 of 0.6 was reached. The cultures were centrifuged at $4,000 \mathrm{~g}$ at $4^{\circ} \mathrm{C}$ for 10 minutes. For the exogenous addition of protein, cell pellets were resuspended in cold PBS (Bio-Rad) containing $10 \mu \mathrm{M}$ of either ColE1-T or ColE1-TR protein. Cells were incubated with protein with gentle rocking for 1 hour at $37^{\circ} \mathrm{C}$. After incubation with protein, the cells were collected at $4,000 \mathrm{~g}$ at $4{ }^{\circ} \mathrm{C}$ for 10 minutes and resuspended in cold PBS without protein. The centrifugation and wash were then repeated a second time. The PBS washes are designed to remove unbound protein.

Protease digestion of intact cells and lysed cells: Each sample is split into two-1 mL aliquots. The first aliquot is the intact cell condition which is placed on ice. The second aliquot is the lysed cells control for protease activity. This aliquot was lysed by adding $0.25 \mathrm{mg} / \mathrm{mL}$ lysozyme (10 $\mu \mathrm{L}$ of a $25 \mathrm{mg} / \mathrm{mL}$ in $10 \mathrm{mM}$ Tris $\mathrm{pH} 8.0,50 \%(\mathrm{v} / \mathrm{v})$ glycerol) and incubated at room temperature for 15 minutes followed by five freeze-thaw cycles in liquid nitrogen. The intact cells condition and lysed condition were both split into three $270 \mu \mathrm{L}$ aliquots. For each triplet, trypsin was added at 5 and $20 \mu \mathrm{g} / \mathrm{mLs}$ to digest any extracellularly exposed protein. $30 \mu \mathrm{L}$ of PBS was added to the third aliquot of each triplet for a no trypsin sample. The three aliquots were incubated at room temperature to allow for trypsin digestion. After 30 minutes, $20 \mu \mathrm{L}$ of 100 
$\mathrm{mM}$ PMSF was added to terminate the trypsin digestion reaction. Then the samples associated

with the intact cells condition were lysed. Lysozyme was added to a final concentration of 0.25 $\mathrm{mg} / \mathrm{mL}$ and incubated at room temperature for 15 minutes followed by five freeze-thaw cycles in

liquid nitrogen. All samples were stored at $-20{ }^{\circ} \mathrm{C}$ until ready for western-blot analysis.

Western blot: Samples were separated by SDS-PAGE. Colicin proteins were probed with THE HisTag mAb mouse (GenScript). SurA was probed with anti-SurA Rabbit polyclonal (Cusabio). Western blots were imaged by fluorescence using a LI-COR Odyssey imager with secondary antibodies IRDye 800CW goat anti-mouse IgG and IRDye 800CW Donkey anti-Rabbit for Colicins and SurA, respectively.

Single-Molecule Microscopy. Cysteine mutations were introduced at the C-terminus before the histidine tag for fluorophore conjugation. These constructs were purified as described in the Expression and Purification section with the addition of $1 \mathrm{mM}$ TCEP in all buffers. All subsequent steps were performed with limited exposure to light and in amber tubes. Cyanine3 (Cy3) maleimide (Lumiprobe) was reconstituted in DMSO. Fluorophore labeling was achieved by mixing a 20-fold molar excess of $\mathrm{Cy} 3$ maleimide to protein and incubating overnight at $4{ }^{\circ} \mathrm{C}$. Free dye was removed by gel filtration on a Sephadex NAP-10 G-25 column. Simultaneously to the dye removal, the sample was buffer exchanged into storage buffer (PBS pH 7.4, 1 mM DTT, 1 mM EDTA). The degree of labeling was determined spectrophotometrically from the concentrations of the dye and protein solutions using their respective extinction coefficients, $\varepsilon$, as described by their manufacturers or for the proteins as estimated by Expasy ProtParam (Cy3 $\varepsilon_{548 \mathrm{~nm}}=162,000 \mathrm{~L} \mathrm{~mol}^{-1} \mathrm{~cm}^{-1} ;$ ColE1-T-E192C $\varepsilon_{280 \mathrm{~nm}}=9,970 \mathrm{~L} \mathrm{~mol}^{-1} \mathrm{~cm}^{-1} ;$ ColE1-TR-E366C $\left.\varepsilon_{280 \mathrm{~nm}}=14,440 \mathrm{~L} \mathrm{~mol}^{-1} \mathrm{~cm}^{-1}\right)$. Labeling efficiencies were $\sim 75 \%$ and $\sim 85 \%$ for ColE1-T-E192C and ColE1-TR-E366C, respectively. Protein concentrations were adjusted according to the percentage of labeled protein. 
Cultures of $E$. coli (WT, $\Delta$ tolC, or BL21(DE3)) were grown in LB medium at $37{ }^{\circ} \mathrm{C}$ with

shaking (180 r.p.m.) overnight, then transferred to MOPS minimal medium (Teknova) with $0.2 \%$ glycerol and $1.32 \mathrm{mM} \mathrm{K}_{2} \mathrm{HPO}_{4}$ and grown at $37{ }^{\circ} \mathrm{C}$ for $13 \mathrm{~h}$. The sample was transferred to MOPS medium and grown to turbidity at $37^{\circ} \mathrm{C}$ overnight. A $1-\mathrm{mL}$ aliquot of culture was centrifuged for $2 \mathrm{~min}$ at 4,850 g to pellet the cells. The pellet was washed in $1 \mathrm{~mL}$ MOPS and centrifuged a second time. The supernatant was then removed, and the cell pellet was resuspended in $500 \mu \mathrm{L}$ MOPS. A $1.0 \mu \mathrm{L}$ droplet of concentrated cells was placed onto a glass slide. Then, a $1.0 \mu \mathrm{L}$ droplet of $1 \mu \mathrm{g} / \mathrm{mL}$ colicin E1 protein construct stock was added to the cells. The droplet was covered by an agarose pad (1\% agarose in MOPS media) and a second coverslip. Samples were imaged at room temperature using wide-field epifluorescence microscopy with sensitivity to detect single dye molecules as described previously (Tuson \& Biteen, 2015). Briefly, fluorescence was excited by a 561-nm laser (Coherent Sapphire 560-50) for Cy3 or a 488-nm laser (Coherent Sapphire 488-50) for GFP. The lasers were operated at low power densities $\left(1-2 \mathrm{~W} / \mathrm{cm}^{2}\right)$, and fluorescence was imaged with an Olympus IX71 inverted microscope with a 100x, 1.40-NA phase-contrast oil-immersion objective and appropriate excitation, emission, and dichroic filters. A Photometrics Evolve electron multiplying chargecoupled device (EMCCD) camera with > 90\% quantum efficiency captured the images at a rate of 20 frames per second. Each detector pixel corresponds to a $49 \mathrm{~nm} \times 49 \mathrm{~nm}$ area of the sample. $E$. coli cells were identified and fluorescent signals were detected using the Single-Molecule Localization by Local Background Subtraction (SMALL-LABS) algorithm. (Isaacoff et al., 2019)

Co-elution. The interaction of TolC and colicin E1-T or -TR were determined by co-elution on an SEC column. Purified TolC and colicin E1-T or -TR were mixed at a 1:2 molar ratio to ensure an excess of colicin to saturate TolC binding sites and incubated at room temperature for 1 hour before loading onto a Superdex 200 Increase 10/300 GL column (GE Healthcare). The protein 
581 was eluted with 1.5 column volumes into $20 \mathrm{mM}$ Tris $\mathrm{pH} 8.0,40 \mathrm{mM} \mathrm{NaCl}, 0.05 \%$ n-dodecyl- $\beta$ -

582 D-maltoside for ColE1-T. For ColE1-TR the $\mathrm{NaCl}$ concentration was increased to $200 \mathrm{mM}$ to

583 prevent precipitation. Elution fractions were collected every $0.5 \mathrm{~mL}$. Peak fractions were

584 concentrated to $20 \mu \mathrm{L}$ and analyzed by $4-20 \%$ SDS-PAGE.

Real-Time Efflux. Real-time efflux activity in the presence of ColE1-TR was determined as 587 previously described with some modifications (Bohnert et al., 2010, Bohnert et al., 2011). Cells 588 were resuspended to OD600 1.5 in cold PBS with and without $10-100 \mu \mathrm{M}$ colicin proteins and 589 incubated for 15 minutes on ice. To turn off efflux, $100 \mu \mathrm{M}$ carbonyl cyanide m-chlorophenyl 590 hydrazone (CCCP) was added. After an additional 15 minutes the efflux dye NNN was added to 591 the cells to $10 \mu \mathrm{M}$. To determine cell viability after treatment with CCCP and NNN, we counted 592 the colony-forming units of treated and untreated cells. Treated cells had $93 \%$ viability compared 593 to untreated cells as determined by colony-forming units (treated cells had $4.4 \times 10^{9} \mathrm{CFU} / \mathrm{mL}$, 594 untreated cells had $\left.4.7 \times 10^{9} \mathrm{CFU} / \mathrm{mL}\right)$. The cells were incubated at $25^{\circ} \mathrm{C}$ with shaking at 140 595 r.p.m. for 2 hours. Cells were harvested at 3,500 g for 5 minutes and washed once in $20 \mathrm{mM}$ 596 potassium phosphate buffer $\mathrm{pH} 7$ with $1 \mathrm{mM} \mathrm{MgCl}_{2}$. Cell concentrations were adjusted to OD600 $597 \quad 1.0$ and placed on ice. Then, $2 \mathrm{~mL}$ of the cell suspension was loaded into a quartz cuvette (Agilent 598 Technologies). Fluorescence was measured with an Agilent Cary Eclipse fluorescence 599 spectrophotometer with slit widths at 5 and $10 \mathrm{~nm}$ for excitation wavelength of $370 \mathrm{~nm}$ and 600 emission wavelength of $415 \mathrm{~nm}$. Fluorescence measurements were taken every 1 second. After 601100 seconds, $50 \mathrm{mM}$ glucose was added to re-energize the cells and initiate efflux, and 602 fluorescence data were collected for an additional 600 seconds. Figure 5A-C, reflects time after 603 glucose addition. 
605 Minimum Inhibitory Concentrations (MICs). MICs were determined using the broth dilution method (Wiegand et al., 2008) with some modifications in 96 well plate format using LB media

\section{7} in $100 \mu \mathrm{L}$ well volumes. Cultures were grown at $37{ }^{\circ} \mathrm{C}$ with shaking at 250 r.p.m. and OD600 was read on a Biotek plate reader after 20 hours. MICs are defined by the lowest concentration that prevents visible growth. We chose an OD600 of $>0.1$ as the cutoff for growth. We report MICs as the mean of 3 biological replicates with each replicate plotted (Figure 5D, Figure 5, source data 1). Due to the 2 -fold discretized nature of concentration ranges used to determine MICs we do not report statistical significance values as is typical of MIC reporting.

Reconstitution of TolC into Amphipol. $1 \mathrm{~mL}$ of TolC at $0.5 \mathrm{mg} / \mathrm{mL}$ was mixed with $0.75 \mathrm{~mL}$ of A8-35 (Anatrace) amphipol at $33 \mathrm{mg} / \mathrm{mL}$ for a 50 -fold weight excess and incubated at room temperature for 30 minutes. Bio-Beads SM-2 resin that was washed in methanol and equilibrated with $20 \mathrm{mM}$ Tris, $40 \mathrm{mM} \mathrm{NaCl}$ was added to the reaction mixture at $0.5 \mathrm{~g} / \mathrm{mL}$ to initiate detergent exchange for A8-35 and incubated with rotation at $4{ }^{\circ} \mathrm{C}$ overnight. The mixture was transferred to a tube with fresh Bio-Beads and incubated at $4{ }^{\circ} \mathrm{C}$ for an additional 4 hours. The reaction mixture was loaded onto a HiLoad 16/60 Superdex 200 pg column on an ÄKTA Pure FPLC system and eluted with 1.5 column volumes in $20 \mathrm{mM}$ Tris $\mathrm{pH} 8.0,40 \mathrm{mM} \mathrm{NaCl}$ to remove free A8-35 and detergent. For colicin-bound TolC in A8-35, colicin E1-T was added to the reaction mixture at a $>2$ molar excess prior to gel filtration. TolC or colicin-bound TolC in A8-35 was concentrated to $2-4 \mathrm{mg} / \mathrm{mL}$ for cryoEM.

\section{Reconstitution of TolC into lipid nanodiscs. POPC (Avanti Polar Lipids) in chloroform was} dried under a stream of nitrogen and freeze dried to remove residual chloroform. Lipids were reconstituted to $50 \mathrm{mM}$ in cholate buffer $(20 \mathrm{mM}$ Tris $\mathrm{pH} 8.0,100 \mathrm{mM} \mathrm{NaCl}, 0.5 \mathrm{mM}$ EDTA, $100 \mathrm{mM}$ cholate) in an amber glass vial. The vial was submerged under a stream of warm water 
until the solution became clear. Lipids, membrane scaffold protein, and TolC were mixed in a 36:1:0.4 ratio as previously described (Daury et al., 2016). Final concentrations were $4.5 \mathrm{mM}$

\section{2} POPC, $125 \mu \mathrm{M}$ MSP1D1, $50 \mu \mathrm{M}$ TolC in a $2 \mathrm{~mL}$ reaction with cholate brought up to $20 \mathrm{mM}$ and dodecyl-maltoside up to $0.1 \%$. The reaction mixture was incubated on ice for 1 hour. Bio-Beads SM-2 resin that was washed in methanol and equilibrated with $20 \mathrm{mM}$ Tris, $40 \mathrm{mM} \mathrm{NaCl}$ were added to the reaction mixture at $0.5 \mathrm{~g} / \mathrm{mL}$ to initiate nanodisc formation and incubated with rotation at $4^{\circ} \mathrm{C}$ overnight. The mixture was transferred to a tube with fresh Bio-Beads and incubated at $4{ }^{\circ} \mathrm{C}$ for an additional 4 hours. The reaction mixture was loaded onto a HiLoad 16/60 Superdex 200 pg column on an ÄKTA Pure FPLC system and eluted with 1.5 column volumes in $20 \mathrm{mM}$ Tris $\mathrm{pH}$ 8.0, $40 \mathrm{mM} \mathrm{NaCl}$ to separate TolC inserted into nanodiscs from empty nanodiscs. For colicin-bound TolC in nanodiscs, colicin E1-T was added to the reaction mixture at a $>2$ molar excess prior to gel filtration. TolC or colicin-bound TolC in nanodiscs was concentrated to 2-4 mg/mL for cryoEM.

\section{Cryoelectron microscopy. $3 \mu \mathrm{L}$ of protein solution (TolC/ColE1-T in amphipol or TolC/ColE1-} $\mathrm{T}$ in nanodiscs) was diluted to approximately $1.05 \mathrm{mg} / \mathrm{mL}$ concentration, applied to a glowdischarged TEM grid, and plunge-frozen in ethane using a Vitrobot Mark IV (FEI Company) with grade 595 filter paper (Ted Pella). Glow discharge was performed in ambient atmosphere at 0.39 mBar pressure. Imaging was performed using a Talos Arctica (FEI Company) operated at $200 \mathrm{kV}$ with energy-filter and K2 Summit (Gatan, Inc.) for detection. To collect multiple images per hole while maintaining parallel illumination conditions, a nonstandard $20 \mu \mathrm{m}$ condenser aperture was used to image TolC-ColE1-T in nanodiscs. At nominal magnification of 205,000×, images were acquired in counting mode with a calibrated pixel size of $0.6578 \AA$. Fresnel fringes attributable to the beam interaction with the aperture were often seen in images. Some investigators have moved the microscope stage and altered the nominal objective lens true focus point to generate a fringe- 
655 free condition (Weis \& Hagen, 2020). In this study, imaging at $205,000 \times$ with a $20 \mu \mathrm{m}$ aperture yielded better results than imaging at $130,000 \times$ with a $50 \mu \mathrm{m}$ aperture; at $130,000 \times$ with a $20 \mu \mathrm{m}$ aperture the fringes were extremely severe due to the larger field of view, so a full dataset was not collected with those conditions. TolC in nanodiscs (without ColE1-T) was imaged at 130,000x with a $50 \mu \mathrm{m}$ condenser aperture (Figure 1 , source data 1$)$.

Micrographs were collected with SerialEM (Mastronarde, 2018) using in-house modifications of the scripts of Chen Xu (sphinx-emdocs.readthedocs.io). Briefly, multishot imaging was configured with 4 images per hole for each of 16 holes; intermediate-magnification montages of grid squares were acquired; points were selected manually for collection of 64 images per point; images were acquired using coma-compensated image shift as gain- and darkcorrected LZW-compressed TIFs. Side, top, and oblique views were seen in areas of thin ice. During screening, ice thickness was estimated at $17-30 \mathrm{~nm}$ by the method of $I_{0} / I_{\mathrm{ZLP}}$ (Rice et al., 2018).

The collection of micrographs of TolC without colicin at 130,000× magnification has been previously described (Budiardjo et al., 2021).

3D reconstruction and modeling. Final reconstructions were obtained using cryoSPARC 2 (Punjani et al., 2017). 1,018 micrographs were collected of amphipol-embedded TolC/ColE1-T. Micrographs were motion-corrected using RELION 3 (Zivanov et al., 2018). CTF parameters were determined by means of ctffind (Rohou \& Grigorieff, 2015). 115,362 particles were selected with crYOLO (Wagner et al., 2019). 2D classification revealed that many particles had aberrant morphology and only $24,624(21 \%)$ were selected for 3D reconstruction. Ab initio reconstruction in cryoSPARC 2 (Punjani et al., 2017) was effective at recovering a map whose shape was similar to that of previously described TolC trimers. However, the data could only be refined to a nominal global resolution of $6.0 \AA$, and lumenal density was insufficiently resolved. 4,492 micrographs were then collected of nanodisc-embedded TolC/ColE1-T and processed similarly. 
680 Of the 339,779 particles detected by cryoSPARC Live, 179,834 (53\%) were in good classes. Although there were slightly fewer particles per micrograph in the nanodisc dataset, more

\section{2}

particles per micrograph were usable. Beginning with the ab initio model and mask derived from the amphipol data, this particle set was refined by cryoSPARC 2 non-uniform refinement with or without imposed $\mathrm{C}_{3}$ symmetry. The maps refined to nominal global resolutions of $2.81 \AA$ and $3.09 \AA$ for the symmetrized and asymmetric maps, respectively. There was local variation in resolution within the map, with consistent, high resolution in the middle of TolC and lower resolution at the lids and for ColE1-T. Local resolution was computed in cryoSPARC by the locally windowed FSC method (Cardone et al., 2013) and rendered with UCSF Chimera. To reduce the voxel-based values to averages for four regions of the complex, the local resolution map was masked to include only voxels within $3 \AA$ of a modeled atom and then the median value was calculated for those voxels closest to ColE1, closest to TolC residues 168-172 and 386-390, closest to TolC residues 285-301 and 76-82, or closest to other TolC residues. Furthermore, it is notable that while the nanodisc appears as a double-belt in the symmetrized map, in the asymmetric map the nanodisc protein mostly appears on the side of TolC that is bound to ColE1T. One possible explanation is that, despite masking, nanodisc asymmetry is a confounder of the asymmetric refinement and is one source of heterogeneity in the data. Another possibility is that the C-terminus of ColE1-T forms an interaction with the nanodisc, causing preferential alignment of the nanodisc with respect to the TolC/ColE1-T complex.

196,158 particles of TolC without colicin were obtained as previously described (Budiardjo et al., 2021). Homogeneous refinement yielded a structure at $2.89 \AA$; local motion correction and global CTF refinement yielded a final map at $2.84 \AA$.

Modeling was initiated by rigidly docking a crystal structure of TolC in complex with hexamminecobalt (1TQQ) (Higgins et al., 2004) into the symmetrized map density. Automated, semi-automated, and manual real-space refinement was performed using phenix (Afonine et al., 
2018), ISOLDE (Croll, 2018), and coot (Emsley et al., 2010). For TolC with ColE1-T, additional refinement was performed in AMBER using the cryoEM density map as a restraint. Although additional residues are present at the TolC C-terminus, these were not modeled because the density becomes unsharp after residue 428. Blurred density in the map suggests that the Cterminus follows helix 3 towards the periplasmic end of the molecule. ColE1-T was modeled $a b$ initio. The 3-fold symmetrized map contains density at $\sim 1 / 3^{\text {rd }}$ occupancy for ColE1-T and this density contains some high-resolution information not present in the asymmetric map, except near the TolC lid regions where symmetrization overlays ColE1-T density with TolC density at a threefold-related position. First, polyalanine helices were placed in the helical density in coot. Cross-correlation coefficients for both helices are higher with the N-terminus oriented towards the periplasm. Moreover, the side chains angle slightly towards the N-terminus like the fronds of an evergreen angle towards the ground in typical "Christmas tree" conformation, indicating that this is the correct chain orientation. An estimate of the register was made by visual inspection of potential anchor residues. Finally, the hinge region was filled in using phenix and coot. This completed chain was refined against the asymmetric map in ISOLDE. Iteration between phenix, coot, and ISOLDE was continued until acceptable fit to density was achieved. In the case of TolC with ColE1-T, the map was further improved by combining map information with molecular dynamics force fields (Wu et al., 2013). Briefly, starting with the phenix/coot/ISOLDE-refined model, we performed restrained simulated annealing in AMBER, heating from $0 \mathrm{~K}$ to $300 \mathrm{~K}$ for $0.2 \mathrm{nsec}$, holding constant temperature for $1 \mathrm{nsec}$, and then cooling to $0 \mathrm{~K}$ over $0.2 \mathrm{nsec}$. The cryoEM density map is utilized as a restraint potential in the annealing so that both map information and AMBER force field information are simultaneously utilized to obtain an optimum model consistent with the data (Wu et al., 2013). The protein force field used the ff14SB force field(Maier et al., 2015) and a generalized Born implicit solvent model with igb=8 (Nguyen et al., 2013), and a nonbonded cutoff of $20 \AA$. The relative weight of real-space map-based 
restraints and the force field was fixed using $f$ cons $=0.02$. For ColE1-T, information from the symmetrized map was integrated into the modeling procedure during manual remodeling in coot, but map-based refinement in phenix, ISOLDE, and AMBER were against the asymmetric map. TolC without ColE1-T was modeled similarly but using the TolC-ColE1-T structure as a starting point instead of 1TQQ, and without final AMBER refinement.

Molecular representations were generated with Chimera, ChimeraX (Goddard et al., 2018) or PyMOL (Schrödinger, LLC).

\section{AlphaFold model of ColE1}

AlphaFold (Jumper et al., 2021) was used to generate a predicted structural model for full-length ColE1. The amino acid sequence for residues 1-522 of ColE1 was submitted to the AlphaFold Colab

(https://colab.research.google.com/github/deepmind/alphafold/blob/main/notebooks/AlphaFold.ip ynb) with the 'prokaryotic' parameter set as 'True'.

\section{References}

Afonine, P. V., Poon, B. K., Read, R. J., Sobolev, O. V., Terwilliger, T. C., Urzhumtsev, A. \& Adams, P. D. (2018). Acta Crystallogr D Struct Biol 74, 531-544.

Al-Husini, N., Tomares, D. T., Pfaffenberger, Z. J., Muthunayake, N. S., Samad, M. A., Zuo, T., Bitar, O., Aretakis, J. R., Bharmal, M. M., Gega, A., Biteen, J. S., Childers, W. S. \& Schrader, J. M. (2020). Mol Cell 78, 670-682 e678.

Alexander, C. \& Rietschel, E. T. (2001). Journal of Endotoxin Research 7, 167-202.

Bakshi, S., Siryaporn, A., Goulian, M. \& Weisshaar, J. C. (2012). Mol Microbiol 85, 21-38.

Barad, B. A., Echols, N., Wang, R. Y., Cheng, Y., DiMaio, F., Adams, P. D. \& Fraser, J. S. (2015). Nat Methods 12, 943-946.

Behrens, H. M., Lowe, E. D., Gault, J., Housden, N. G., Kaminska, R., Weber, T. M., Thompson, C. M. A., Mislin, G. L. A., Schalk, I. J., Walker, D., Robinson, C. V. \& Kleanthous, C. (2020). mBio 11.

Benedetti, H., Frenette, M., Baty, D., Knibiehler, M., Pattus, F. \& Lazdunski, C. (1991). J Mol Biol 217, 429-439.

Benedetti, H., Frenette, M., Baty, D., Lloubès, R., Geli, V. \& Lazdunski, C. (1989). Microbiology 135, 3413-3420.

Benedetti, H., Lloubes, R., Lazdunski, C. \& Letellier, L. (1992). EMBO J 11, 441-447.

Besingi, R. N. \& Clark, P. L. (2015). Nat Protoc 10, 2074-2080.

Bohnert, J. A., Karamian, B. \& Nikaido, H. (2010). Antimicrob Agents Chemother 54, 3770-3775. 
Bohnert, J. A., Schuster, S., Szymaniak-Vits, M. \& Kern, W. V. (2011). PLoS One 6, e21196. Brunden, K. R., Uratani, Y. \& Cramer, W. A. (1984). The Journal of biological chemistry 259, $7682-7687$.

Buchanan, S. K., Lukacik, P., Grizot, S., Ghirlando, R., Ali, M. M., Barnard, T. J., Jakes, K. S., Kienker, P. K. \& Esser, L. (2007). EMBO J 26, 2594-2604.

Budiardjo, S. J., Ikujuni, A. P., Firlar, E., Cordova, A., Kaelber, J. T. \& Slusky, J. S. G. (2021). J. Membrane Biol.

Cardone, G., Heymann, J. B. \& Steven, A. C. (2013). J Struct Biol 184, 226-236.

Cascales, E., Buchanan, S. K., Duché, D., Kleanthous, C., Lloubès, R., Postle, K., Riley, M., Slatin, S. \& Cavard, D. (2007). Microbiology and Molecular Biology Reviews 71, 158229.

Chavent, M., Duncan, A. L., Rassam, P., Birkholz, O., Helie, J., Reddy, T., Beliaev, D., Hambly, B., Piehler, J., Kleanthous, C. \& Sansom, M. S. P. (2018). Nat Commun 9, 2846.

Cramer, W. A., Sharma, O. \& Zakharov, S. D. (2018). Biochemical Journal 475, 3903-3915.

Croll, T. I. (2018). Acta Crystallogr D Struct Biol 74, 519-530.

Dankert, J., Hammond, S. M. \& Cramer, W. A. (1980). J Bacteriol 143, 594-602.

Dankert, J. R., Uratani, Y., Grabau, C., Cramer, W. A. \& Hermodson, M. (1982). J Biol Chem 257, 3857-3863.

Daury, L., Orange, F., Taveau, J. C., Verchere, A., Monlezun, L., Gounou, C., Marreddy, R. K., Picard, M., Broutin, I., Pos, K. M. \& Lambert, O. (2016). Nat Commun 7, 10731.

de Zamaroczy, M., Mora, L., Lecuyer, A., Geli, V. \& Buckingham, R. H. (2001). Mol Cell 8, 159168.

Dhar, R., Feehan, R. \& Slusky, J. S. G. (2021). J Phys Chem B 125, 3622-3628.

Duche, D. (2007). J Bacteriol 189, 4217-4222.

Eisenberg, D., Weiss, R. M., Terwilliger, T. C. \& Wilcox, W. (1982). Faraday Symposia of the Chemical Society 17, 109-120.

Elkins, P., Bunker, A., Cramer, W. A. \& Stauffacher, C. V. (1997). Structure 5, 443-458.

Emsley, P., Lohkamp, B., Scott, W. G. \& Cowtan, K. (2010). Acta Crystallogr D Biol Crystallogr 66, 486-501.

Escuyer, V. \& Mock, M. (1987). Mol Microbiol 1, 82-85.

Francis, M.-L. R., Webby, M. N., Housden, N. G., Kaminska, R., Elliston, E., Chinthammit, B., Lukoyanova, N. \& Kleanthous, C. (2021). The EMBO Journal 40, e108610.

Franklin, M. W., Nepomnyachiy, S., Feehan, R., Ben-Tal, N., Kolodny, R. \& Slusky, J. S. G. (2018). Structure 26, 1266-1274.e1262.

Freeman, J. T. C. \& Wimley, W. C. (2012). Bioinformatics 28, 2425-2430.

Gerding, M. A., Ogata, Y., Pecora, N. D., Niki, H. \& de Boer, P. A. (2007). Mol Microbiol 63, 1008-1025.

Goddard, T. D., Huang, C. C., Meng, E. C., Pettersen, E. F., Couch, G. S., Morris, J. H. \& Ferrin, T. E. (2018). Protein Sci 27, 14-25.

Hagn, F., Nasr, M. L. \& Wagner, G. (2018). Nat Protoc 13, 79-98.

Higgins, M. K., Eswaran, J., Edwards, P., Schertler, G. F., Hughes, C. \& Koronakis, V. (2004). J Mol Biol 342, 697-702.

Housden, N. G., Hopper, J. T., Lukoyanova, N., Rodriguez-Larrea, D., Wojdyla, J. A., Klein, A., Kaminska, R., Bayley, H., Saibil, H. R., Robinson, C. V. \& Kleanthous, C. (2013). Science 340, 1570-1574.

Housden, N. G., Loftus, S. R., Moore, G. R., James, R. \& Kleanthous, C. (2005). Proc Natl Acad Sci U S A 102, 13849-13854.

Housden, N. G., Rassam, P., Lee, S., Samsudin, F., Kaminska, R., Sharp, C., Goult, J. D., Francis, M. L., Khalid, S., Bayley, H. \& Kleanthous, C. (2018). Biochemistry 57, 4374-4381. 
Housden, N. G., Webby, M. N., Lowe, E. D., El-Baba, T. J., Kaminska, R., Redfield, C., Robinson, C. V. \& Kleanthous, C. (2021). Nature Communications 12, 4625.

Isaacoff, B. P., Li, Y., Lee, S. A. \& Biteen, J. S. (2019). Biophysical Journal 116, 975-982.

Iyer, R., Ferrari, A., Rijnbrand, R. \& Erwin, A. L. (2015). Antimicrob Agents Chemother 59, 2388-2397.

Jakes, K. S. (2017). J Bacteriol 199.

Jumper, J., Evans, R., Pritzel, A., Green, T., Figurnov, M., Ronneberger, O., Tunyasuvunakool, K., Bates, R., Žídek, A., Potapenko, A., Bridgland, A., Meyer, C., Kohl, S. A. A., Ballard, A. J., Cowie, A., Romera-Paredes, B., Nikolov, S., Jain, R., Adler, J., Back, T., Petersen, S., Reiman, D., Clancy, E., Zielinski, M., Steinegger, M., Pacholska, M., Berghammer, T., Bodenstein, S., Silver, D., Vinyals, O., Senior, A. W., Kavukcuoglu, K., Kohli, P. \& Hassabis, D. (2021). Nature 596, 583-589.

Kamio, Y. \& Nikaido, H. (1976). Biochemistry 15, 2561-2570.

Kaur, H., Jakob, R. P., Marzinek, J. K., Green, R., Imai, Y., Bolla, J. R., Agustoni, E., Robinson, C. V., Bond, P. J., Lewis, K., Maier, T. \& Hiller, S. (2021). Nature 593, 125-129.

Kawabata, T. (2010). Proteins 78, 1195-1211.

Kawabata, T. (2019). Biophys Physicobiol 16, 391-406.

Kawabata, T. \& Go, N. (2007). Proteins 68, 516-529.

Kleanthous, C. (2010). Nat Rev Microbiol 8, 843-848.

Kleanthous, C., Rassam, P. \& Baumann, C. G. (2015). Curr Opin Struct Biol 35, 109-115.

Koronakis, V., Sharff, A., Koronakis, E., Luisi, B. \& Hughes, C. (2000). Nature 405, 914-919.

Kurisu, G., Zakharov, S. D., Zhalnina, M. V., Bano, S., Eroukova, V. Y., Rokitskaya, T. I., Antonenko, Y. N., Wiener, M. C. \& Cramer, W. A. (2003). Nat Struct Biol 10, 948-954.

Lazzaroni, J. C., Fognini-Lefebvre, N. \& Portalier, R. (1989). Mol Gen Genet 218, 460-464.

Loftus, S. R., Walker, D., Mate, M. J., Bonsor, D. A., James, R., Moore, G. R. \& Kleanthous, C. (2006). Proc Natl Acad Sci U S A 103, 12353-12358.

Maier, J. A., Martinez, C., Kasavajhala, K., Wickstrom, L., Hauser, K. E. \& Simmerling, C. (2015). J Chem Theory Comput 11, 3696-3713.

Masi, M., Vuong, P., Humbard, M., Malone, K. \& Misra, R. (2007). J Bacteriol 189, 2667-2676.

Mastronarde, D. N. (2018). Microscopy and Microanalysis 24, 864-865.

Misra, R., Morrison, K. D., Cho, H. J. \& Khuu, T. (2015). J Bacteriol 197, 2479-2488.

Nagel de Zwaig, R. \& Luria, S. E. (1967). J Bacteriol 94, 1112-1123.

Nguyen, H., Roe, D. R. \& Simmerling, C. (2013). J Chem Theory Comput 9, 2020-2034.

Pilsl, H. \& Braun, V. (1995). Mol Microbiol 16, 57-67.

Plate, C. A. \& Luria, S. E. (1972). Proc Natl Acad Sci U S A 69, 2030-2034.

Punjani, A., Rubinstein, J. L., Fleet, D. J. \& Brubaker, M. A. (2017). Nat Methods 14, 290-296.

Rassam, P., Copeland, N. A., Birkholz, O., Toth, C., Chavent, M., Duncan, A. L., Cross, S. J., Housden, N. G., Kaminska, R., Seger, U., Quinn, D. M., Garrod, T. J., Sansom, M. S., Piehler, J., Baumann, C. G. \& Kleanthous, C. (2015). Nature 523, 333-336.

Rassam, P., Long, K. R., Kaminska, R., Williams, D. J., Papadakos, G., Baumann, C. G. \& Kleanthous, C. (2018). Nat Commun 9, 1082.

Rice, W. J., Cheng, A., Noble, A. J., Eng, E. T., Kim, L. Y., Carragher, B. \& Potter, C. S. (2018). J Struct Biol 204, 38-44.

Rohou, A. \& Grigorieff, N. (2015). J Struct Biol 192, 216-221.

Schneider, C. G., Penfold, C. N., Moore, G. R., Kleanthous, C. \& James, R. (1997). Microbiology (Reading) 143 ( Pt 9), 2931-2938.

Seeger, M. A., von Ballmoos, C., Eicher, T., Brandstatter, L., Verrey, F., Diederichs, K. \& Pos, K. M. (2008). Nat Struct Mol Biol 15, 199-205.

Sharma, O., Yamashita, E., Zhalnina, M. V., Zakharov, S. D., Datsenko, K. A., Wanner, B. L. \& Cramer, W. A. (2007). J Biol Chem 282, 23163-23170. 
Shi, Z., Chak, K. F. \& Yuan, H. S. (2005). J Biol Chem 280, 24663-24668.

Storek, K. M., Auerbach, M. R., Shi, H., Garcia, N. K., Sun, D., Nickerson, N. N., Vij, R., Lin, Z., Chiang, N., Schneider, K., Wecksler, A. T., Skippington, E., Nakamura, G., Seshasayee, D., Koerber, J. T., Payandeh, J., Smith, P. A. \& Rutherford, S. T. (2018). Proc Natl Acad Sci U S A 115, 3692-3697.

Studier, F. W., Daegelen, P., Lenski, R. E., Maslov, S. \& Kim, J. F. (2009). J Mol Biol 394, 653680.

Tuson, H. H. \& Biteen, J. S. (2015). Anal Chem 87, 42-63.

Wagner, T., Merino, F., Stabrin, M., Moriya, T., Antoni, C., Apelbaum, A., Hagel, P., Sitsel, O., Raisch, T., Prumbaum, D., Quentin, D., Roderer, D., Tacke, S., Siebolds, B., Schubert, E., Shaikh, T. R., Lill, P., Gatsogiannis, C. \& Raunser, S. (2019). Commun Biol 2, 218.

Weis, F. \& Hagen, W. J. H. (2020). Acta Crystallogr D Struct Biol 76, 724-728.

White, P., Joshi, A., Rassam, P., Housden, N. G., Kaminska, R., Goult, J. D., Redfield, C., McCaughey, L. C., Walker, D., Mohammed, S. \& Kleanthous, C. (2017). Proc Natl Acad Sci U S A 114, 12051-12056.

Wiegand, I., Hilpert, K. \& Hancock, R. E. (2008). Nat Protoc 3, 163-175.

Wiener, M., Freymann, D., Ghosh, P. \& Stroud, R. M. (1997). Nature 385, 461-464.

Williams, C. J., Headd, J. J., Moriarty, N. W., Prisant, M. G., Videau, L. L., Deis, L. N., Verma, V., Keedy, D. A., Hintze, B. J., Chen, V. B., Jain, S., Lewis, S. M., Arendall, W. B., 3rd, Snoeyink, J., Adams, P. D., Lovell, S. C., Richardson, J. S. \& Richardson, D. C. (2018). Protein Sci 27, 293-315.

Wu, X., Subramaniam, S., Case, D. A., Wu, K. W. \& Brooks, B. R. (2013). J Struct Biol 183, 429-440.

Zakharov, S. D., Eroukova, V. Y., Rokitskaya, T. I., Zhalnina, M. V., Sharma, O., Loll, P. J., Zgurskaya, H. I., Antonenko, Y. N. \& Cramer, W. A. (2004). Biophys J 87, 3901-3911.

Zakharov, S. D., Wang, X. S. \& Cramer, W. A. (2016). Biochemistry 55, 5084-5094.

Zivanov, J., Nakane, T., Forsberg, B. O., Kimanius, D., Hagen, W. J., Lindahl, E. \& Scheres, S. H. (2018). Elife 7.

\section{Acknowledgments}

We gratefully acknowledge Daniel Montezano, Pinakin Sukthankar, Rik Dhar, Dwight Deay, Scott Lovell, Matthias Wolf, Alexander Little, Heather Shinogle, and Sarah Noga for discussions and feedback, Mark Richter for the use of his fluorometer, Rajeev Misra for the pTrc vector containing the TolC gene, Vasileios Petrou for guidance on nanodiscs, Chamani Perera for peptide synthesis, Karen Marom for editorial guidance, Batika Saxena for artistic contributions, Joseph Lubin and William Hansen for introductions, and the Office of Advanced Research computing (OARC) at Rutgers for high-performance computing resources.

\section{Funding:}

NIH award R21-GM128022 to JSB

NIGMS awards DP2GM128201, P20GM113117, P20GM103638 and the Gordon and Betty Moore Inventor Fellowship to JSGS, NIGMS awards P20 GM103418 and 2K12GM063651 to SJB.

Competing interests: Authors declare that they have no competing interests. 
Data and materials availability: All data are available in the main text or the supplementary materials." All strains used are commercially available. All plasmids available through Addgene. CryoEM maps and models have been deposited with accession codes EMD-21960, EMD-21959, PDB ID 6WXI, and PDB ID 6WXH. All other data is available in the main text or the supplementary materials.

\section{Supplementary Materials for}

\section{Colicin E1 opens its hinge to plug TolC}

S. Jimmy Budiardjo, Jacqueline J. Stevens, Anna L. Calkins, Ayotunde P. Ikujuni, Virangika K. Wimalasena, Emre Firlar, David A. Case, Julie S. Biteen, Jason T. Kaelber and Joanna S.G. Slusky ${ }^{*}$ 


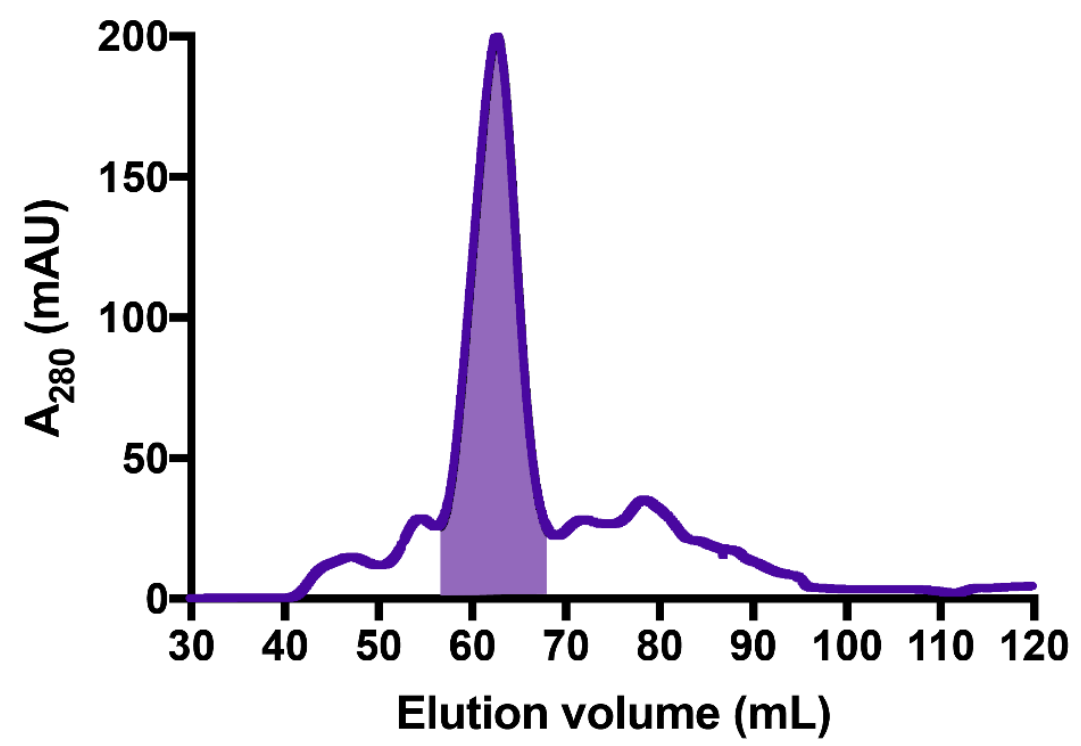

942 Figure 1, supplemental figure 1: Sample SEC chromatogram of TolC purification after 943 refolding. Elution volumes 57 to $68 \mathrm{~mL}$ (shaded) were pooled for further experimentation with 944 TolC. Refolded TolC applied onto a HiLoad 16/60 Superdex 200 pg column on an ÄKTA Pure 945 FPLC system, and eluted with 1.5 column volumes in $20 \mathrm{mM}$ Tris $\mathrm{pH} 8.0,100 \mathrm{mM} \mathrm{NaCl}, 0.05 \%$ 946 n-dodecyl- $\beta$-D-maltoside 


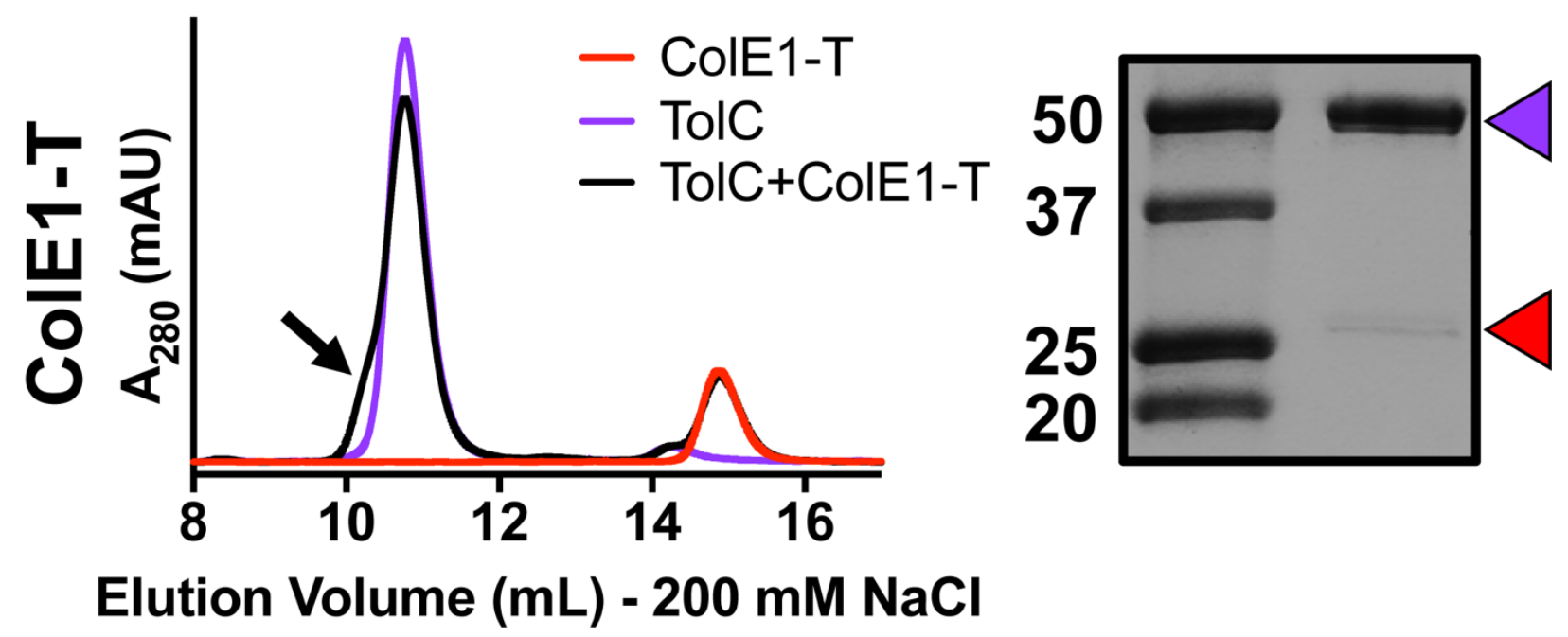

Figure 1 supplemental figure 2. Co-elution of ColE1-T with TolC at $200 \mathrm{mM}$ NaCl (black). Under this higher salt concentration, when TolC (purple) and ColE1-T (red) are mixed, there is a smaller peak shift than that seen with ColE1-TR and the presence of a shoulder (black arrow). SDS-PAGE of the shoulder shows presence of both TolC (purple arrow) and ColE1-T (red arrow). Although some binding was detected, this higher salt concentration prevents full binding as indicated by a much fainter band for ColE1-T than seen at the lower salt concentration (Figure 4A).

Figure 1, source data 1.

Cryo-EM data collection, refinement, and validation statistics 


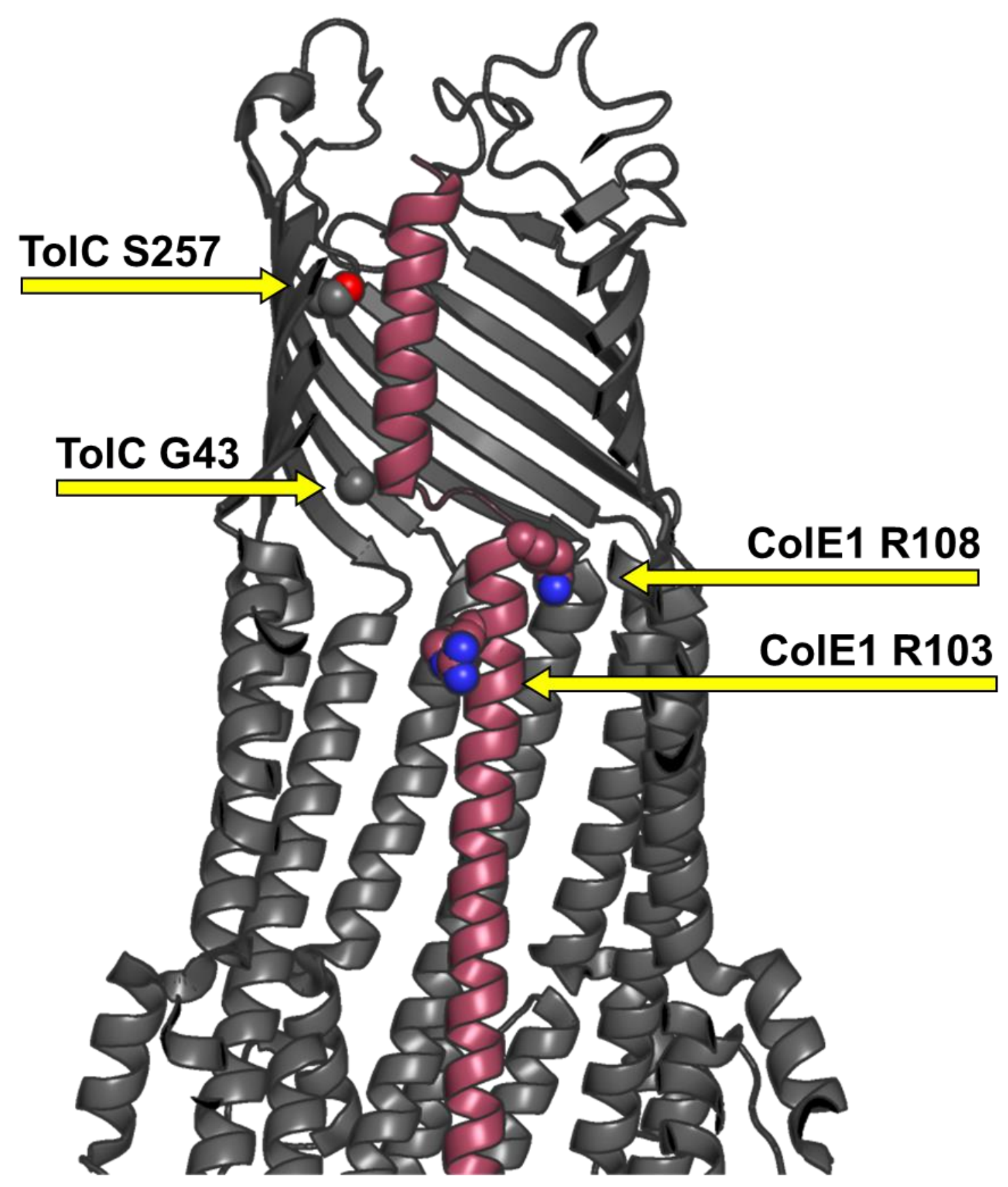

Figure 3, figure supplemental figure 1. Cut away side-view of TolC:ColE1-T complex. Mutations at positions G43 and S257 (spheres) in the TolC barrel (gray) make direct contact with ColE1-T (pink). TolC Mutations to bulkier side chains G43D and S257P were previously shown to abolish WT ColE1 function (Masi et al., 2007) likely due to the introduction of steric clashes that prevent binding. Mutations to ColE1 side chains R108 and R103 (spheres) to glutamine do not abrogate its activity (Jakes, 2017).

Figure 3- video 1. Pan through the atomic model of the TolC/colE1-T complex, from the extracellular aperture to the periplasmic aperture. Carbon atoms of the three TolC polypeptides are colored three different shades of gray and carbon atoms of the colE1-T polypeptide are colored maroon, colE1-T residues of note are colored purple and labeled; field-standard coloring is used for heteroatoms. 


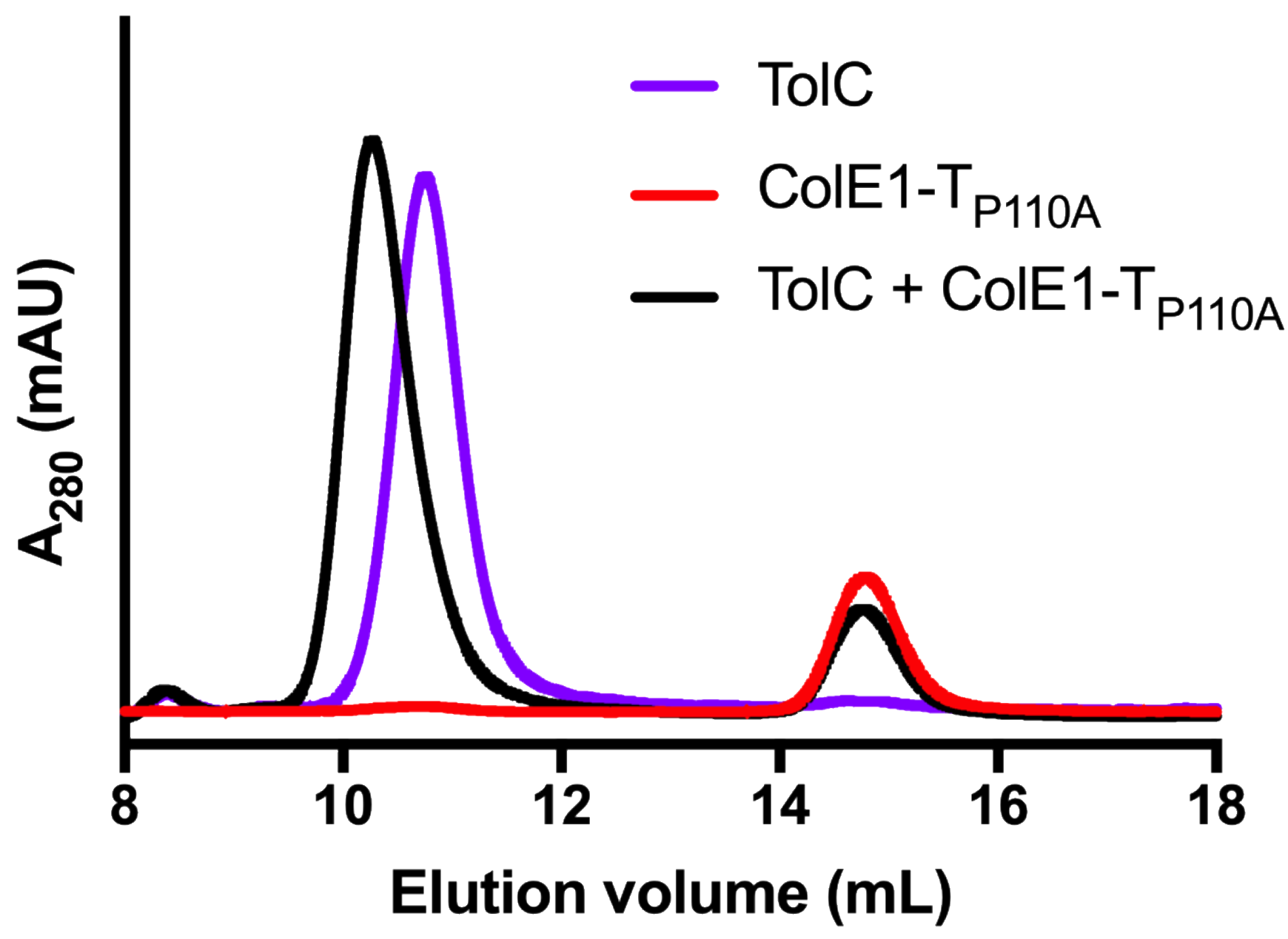

976 Figure 3 supplemental figure 2. ColE1-TP110A retains binding to TolC. SEC chromatogram

977 of ColE1-T $\mathrm{T}_{\mathrm{P} 1 \mathrm{10A}}$ (red line) and TolC (purple line). The arrow indicates the co-elution (black line)

978 fractions (eluted in buffer containing $40 \mathrm{mM} \mathrm{NaCl}$ ) 
WT
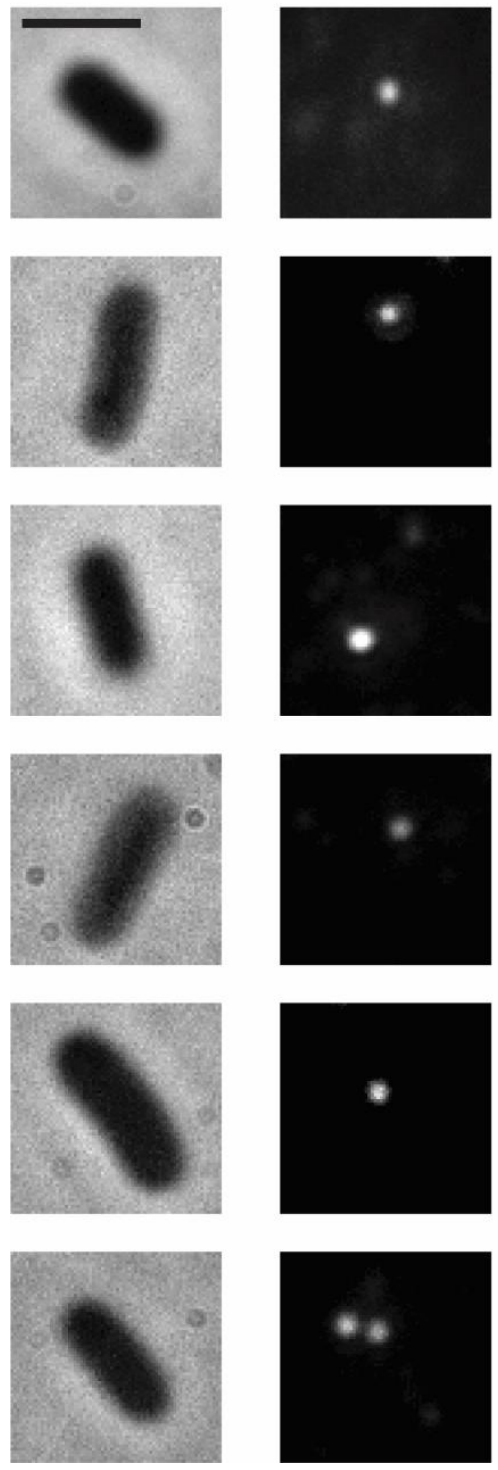

98

$\Delta$ tolC
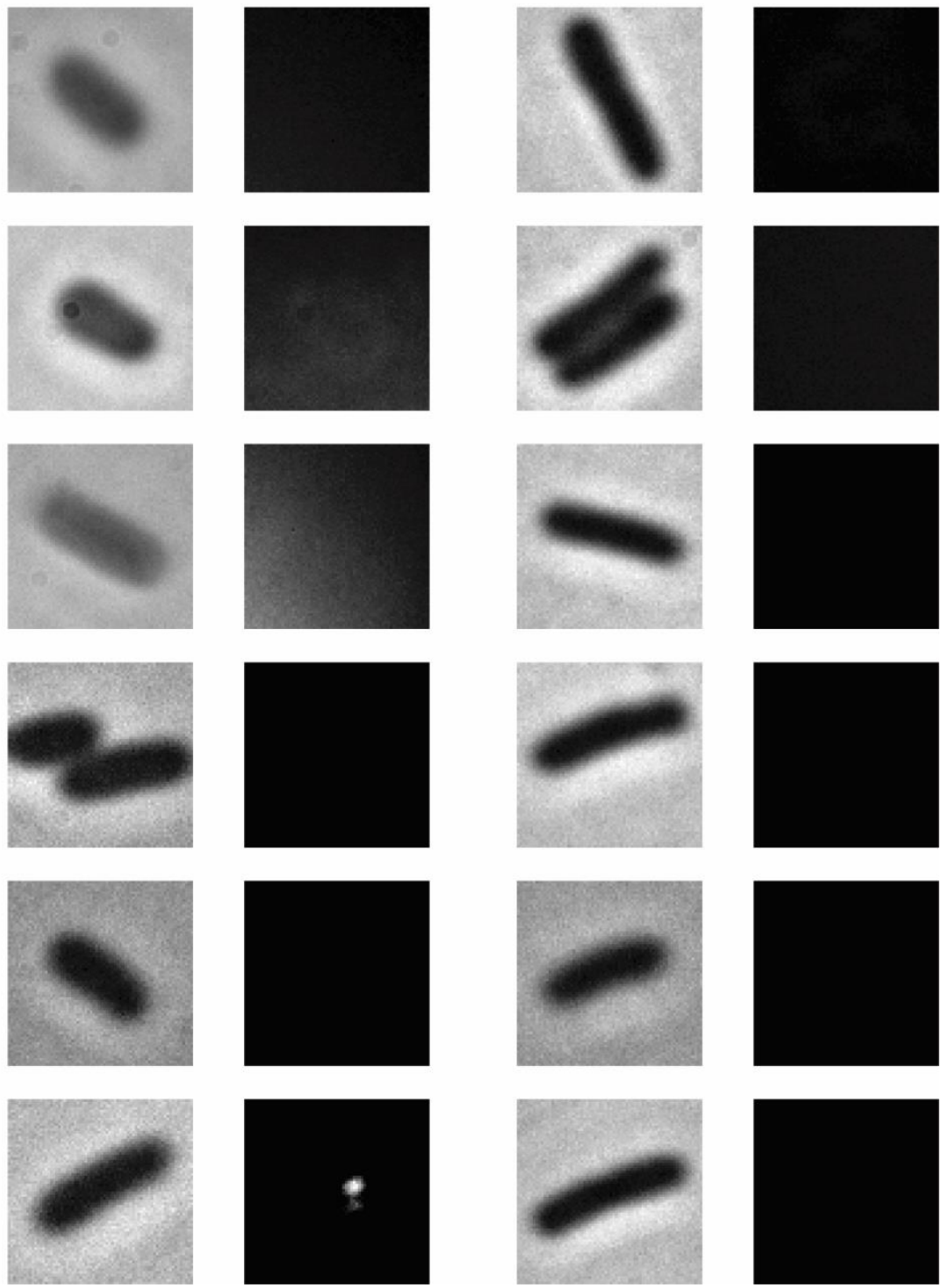

Figure 4 supplemental figure 1: Additional examples of cells observed during imaging described in Figure 4C and D. Brightfield phase-contrast images of living E. coli cells (left) and fluorescence images (right) of ColE1-TR-Cy3 for WT, $\Delta t o l C$, and $\Delta b t u B$. Scale bar: $2 \mu \mathrm{m}$. 

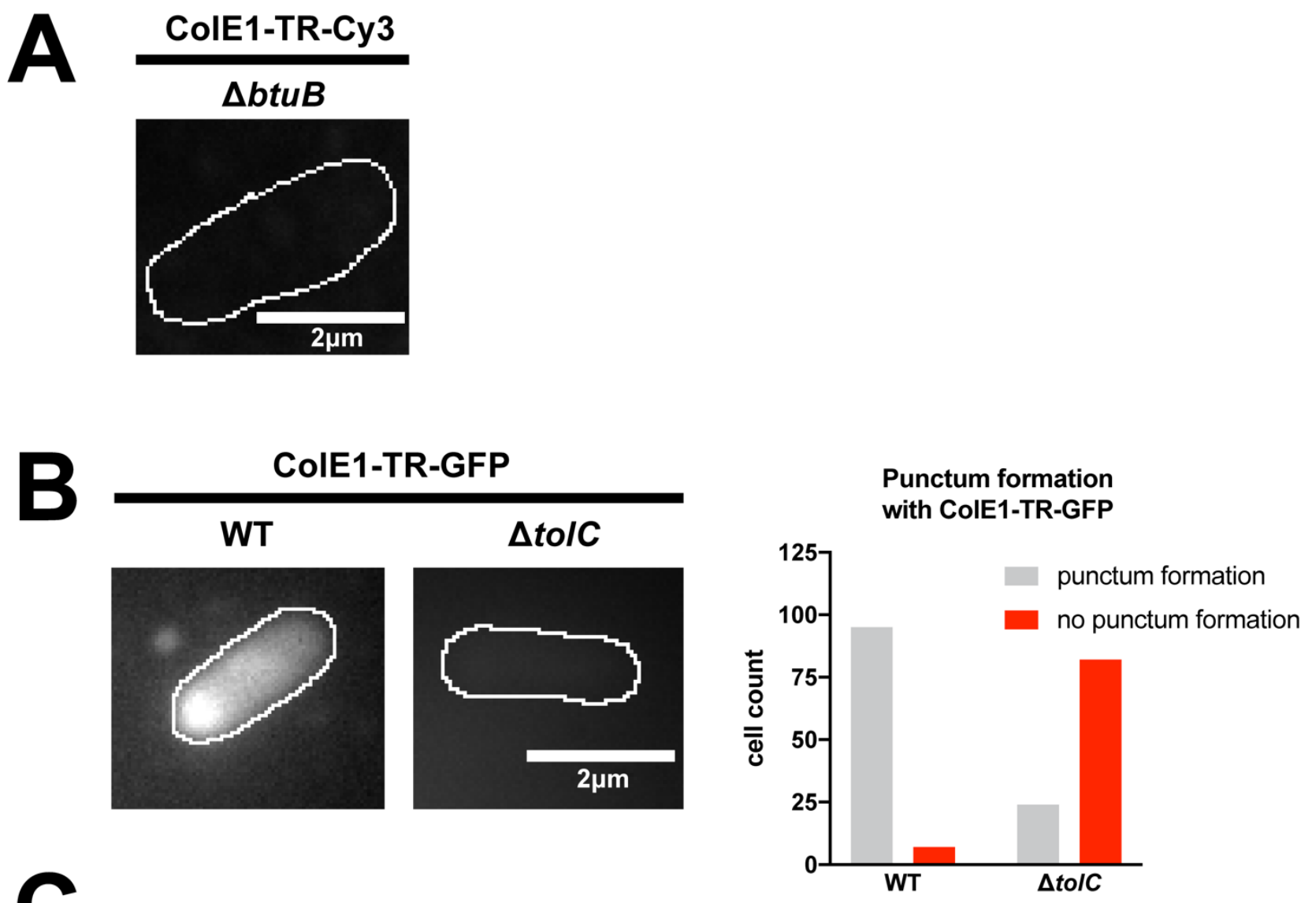

Figure 4 supplemental figure 2: Single-molecule microscopy. Fluorescence images overlaid on outlines of living $E$. coli cells from phase-contrast microscopy for WT and $\Delta$ tolC for ColE1TR-GFP and counts of cells where ColE1-TR-GFP punctum formation was observed. (A) $97 \%$ of cells showed no binding of Cy3-labeled ColE1-TR to $\triangle b t u B$ (B) ColE1-TR-GFP forms similar puncta as Cy3-labeled ColE1-TR (main text Figure 4C). (C) No binding of Cy3-labeled ColE1-T
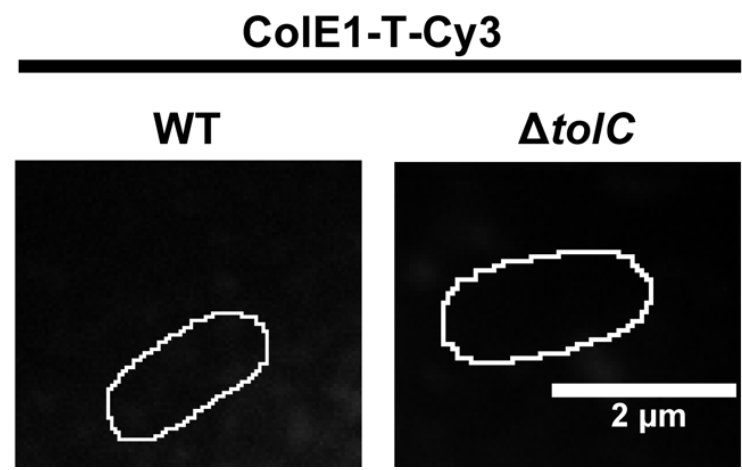
to WT or $\Delta$ tolC cells was detected with Cy3-labeled ColE1-T. 
WT
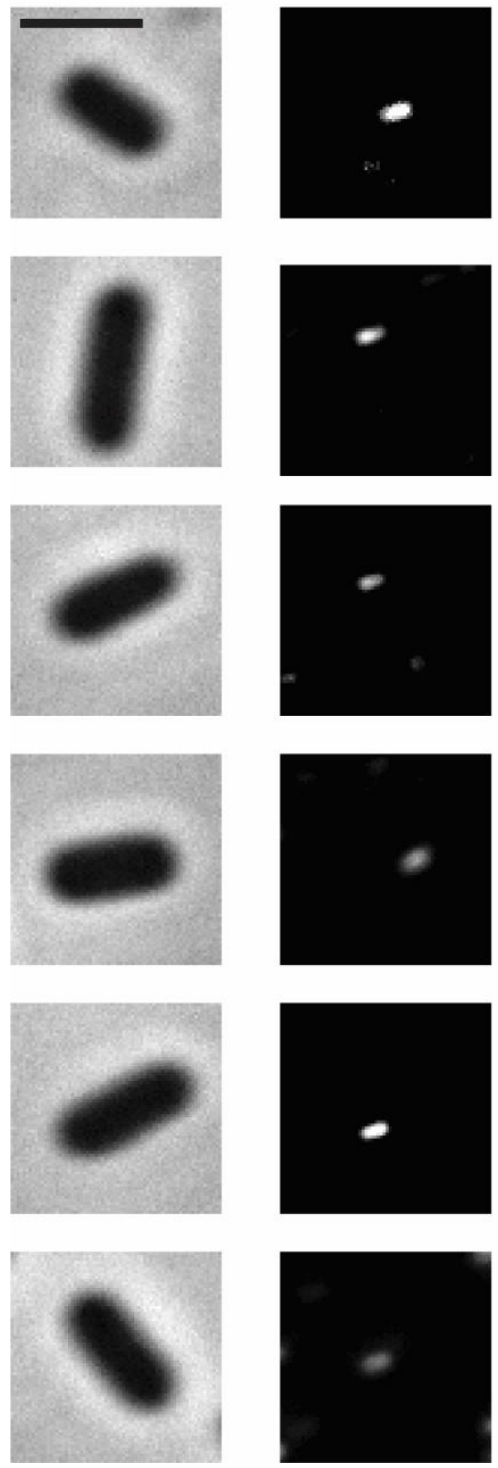

$\Delta$ tolC
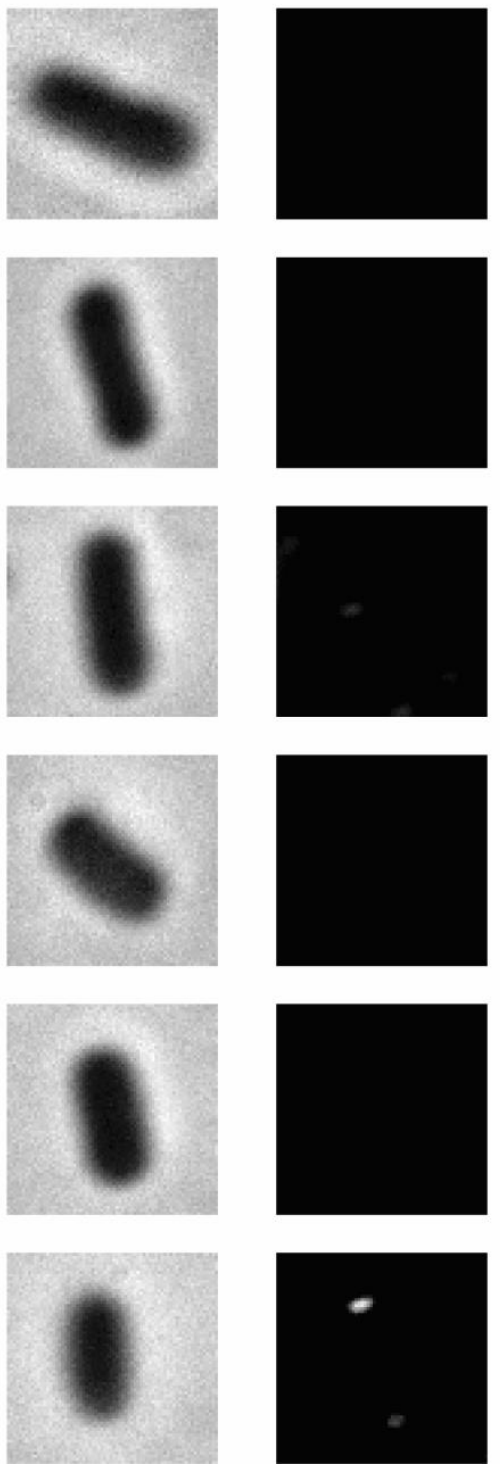

$\Delta b t u B$
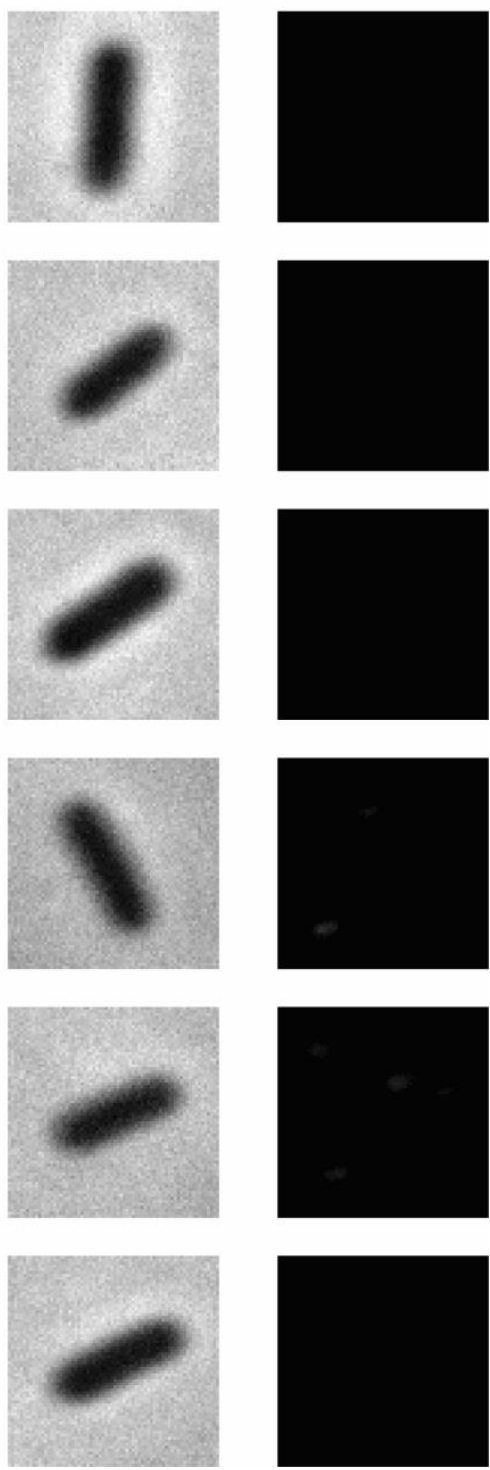

Figure 4 supplemental figure 3: Examples of cells observed with ColE1-TR $\Delta_{1-40}-\mathrm{Cy} 3$.

Brightfield phase-contrast images of living $E$. coli cells (left) and fluorescence images of ColE1-

TR-Cy3 for WT, $\Delta t o l C$, and $\Delta b t u B$. Scale bar: $2 \mu \mathrm{m}$.

Figure 4, source data 1: Comparison of percent cells with puncta and average number of puncta per cell between ColE1-TR-Cy3 and ColE1-TR $\Delta_{1-40}-\mathrm{Cy} 3$ with WT $\triangle$ tolC and $\triangle b t u B$. 


\section{Erythromycin}

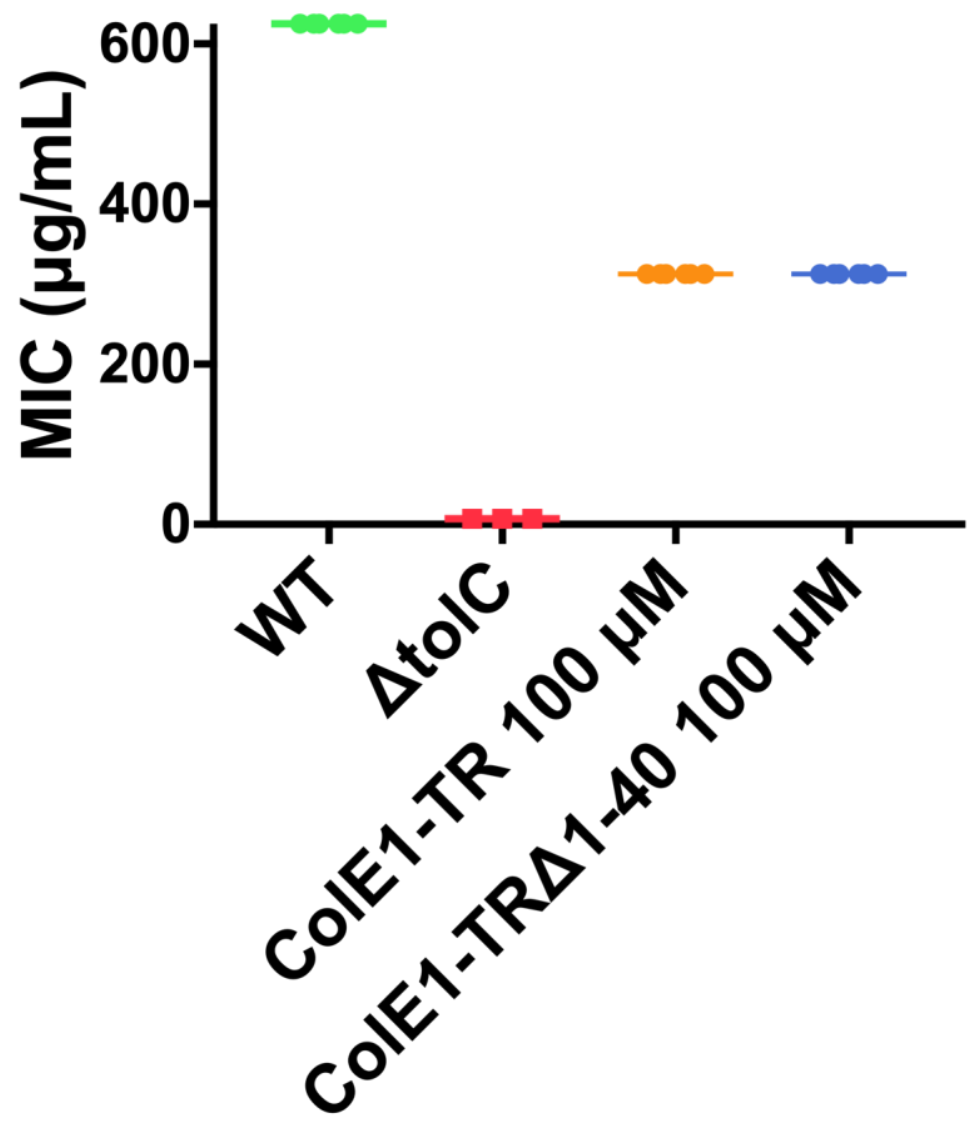

Figure 5 supplemental figure 1. Minimum Inhibitory Concentration of Erythromycin with ColE1-TR TolA box deletion construct. The MIC for erythromycin with $100 \mu \mathrm{M}$ ColE1-TR or ColE1-TR ${ }_{\Delta 1-40}$ is identical indicating that TolA engagement by ColE1-TR does not contribute to antibiotic susceptibility.

Figure 5, source data 1 
Figure 6, supplemental figure 1. ColE1 TR sticks to the surface of $E$. coli but the mechanism of C domain insertion is unclear. The binding between ColE1-R to BtuB and ColE1-T to TolC are likely similar regardless of the presence of the ColE1 C domain. Our work excludes the possibility of the original pillar model. However, because our work did not utilize the $\mathrm{C}$ domain, we cannot distinguish between a modified version of the pillar model and the total thread model.

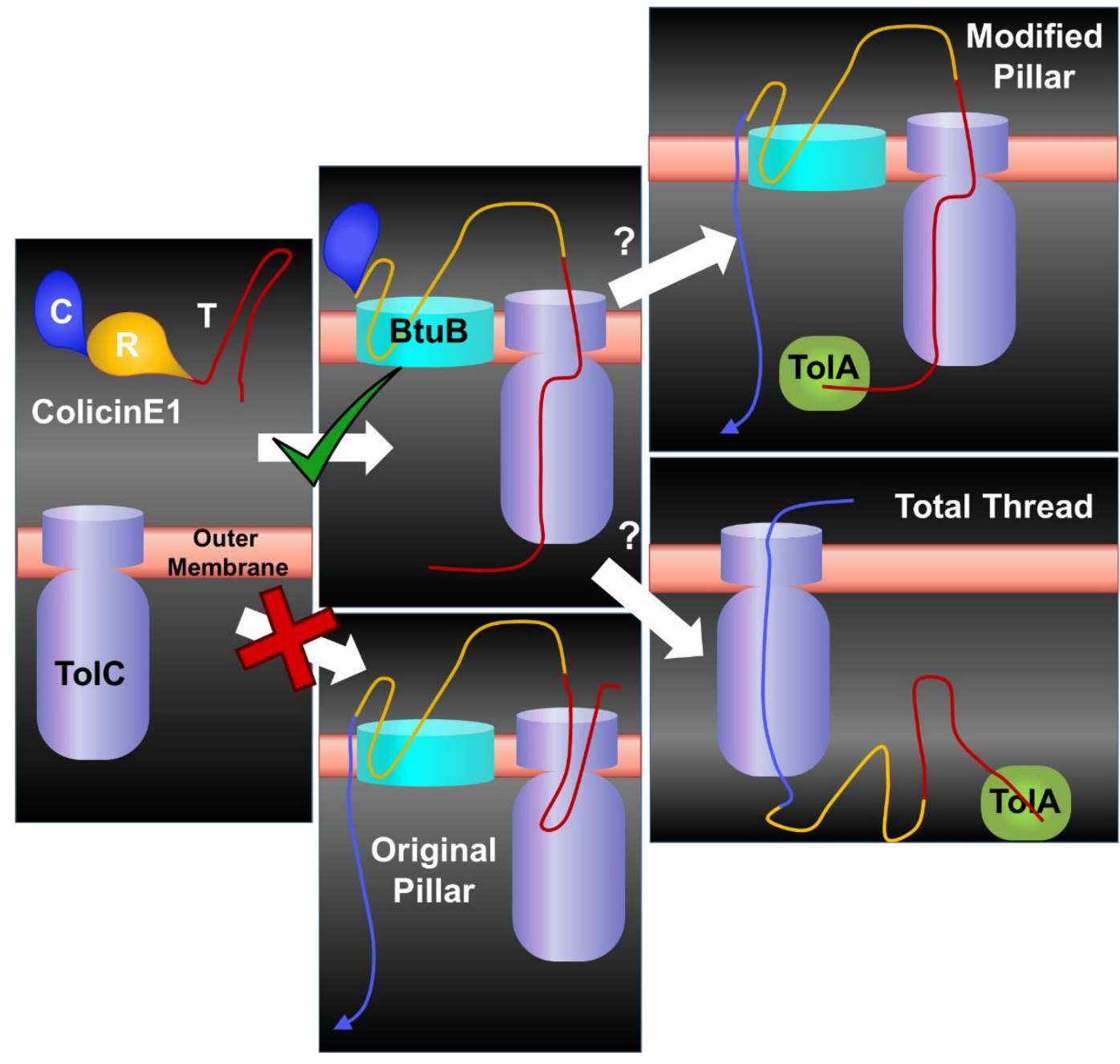




\section{$1027 \quad$ Figure 4 - video 1.}

1028 A representative 30-second movie of ColE1-TR bound to a live cell. ColE1-TR localizes on, and 1029 remains bound to, the extracellular surface of $E$. coli. Fluorescence movie of Cy3-labeled ColE11030 TR on living WT E. coli overlaid on outline of the $E$. coli cell from phase-contrast microscopy.

1031 Continuous imaging at 25 frames per second; the movie plays in real time at the same speed. This 1032 movie corresponds to the first 30 seconds of data used to attain the WT image in Figure 4C. Scale 1033 bar: $2 \mu \mathrm{m}$. 